\title{
Transasymptotics and hydrodynamization of the Fokker-Planck equation for gluons
}

\author{
A. Behtash, ${ }^{1}$ S. Kamata, ${ }^{2}$ M. Martinez® ${ }^{1}{ }^{1}$ T. Schäfer®, ${ }^{1}$ and V. Skokov ${ }^{1,3}$ \\ ${ }^{1}$ Department of Physics, North Carolina State University, Raleigh, North Carolina 27695, USA \\ ${ }^{2}$ National Center for Nuclear Research, PL-00-681 Warsaw, Poland \\ ${ }^{3}$ Riken-BNL Research Center, Brookhaven National Laboratory, Upton, New York 11973, USA
}

(Received 24 November 2020; accepted 10 February 2021; published 15 March 2021)

\begin{abstract}
We investigate the nonlinear transport processes and hydrodynamization of a system of gluons undergoing longitudinal boost-invariant expansion. The dynamics is described within the framework of the Boltzmann equation in the small-angle approximation. The kinetic equations for a suitable set of moments of the one-particle distribution function are derived. By investigating the stability and asymptotic resurgent properties of this dynamical system, we demonstrate, that its solutions exhibit a rather different behavior for large (UV) and small (IR) effective Knudsen numbers. Close to the forward attractor in the IR regime the constitutive relations of each moment can be written as a multiparameter transseries. This resummation scheme allows us to extend the definition of a transport coefficient to the nonequilibrium regime naturally. Each transport coefficient is renormalized by the nonperturbative contributions of the nonhydrodynamic modes. The Knudsen number dependence of the transport coefficient is governed by the corresponding renormalization group flow equation. An interesting feature of the Yang-Mills plasma in this regime is that it exhibits transient non-Newtonian behavior while hydrodynamizing. In the UV regime the solution for the moments can be written as a power-law asymptotic series with a finite radius of convergence. We show that radius of convergence of the UV perturbative expansion grows linearly as a function of the shear viscosity to entropy density ratio. Finally, we compare the universal properties in the pullback and forward attracting regions to other kinetic models including the relaxation time approximation and the effective kinetic Arnold-Moore-Yaffe theory.
\end{abstract}

DOI: 10.1103/PhysRevD.103.056010

\section{INTRODUCTION}

Relativistic fluid dynamics is an effective theory which describes long-wavelength phenomena. It is widely accepted that its regime of validity is restricted to systems near local thermal equilibrium. However, this traditional paradigm has recently been challenged by the overwhelming success of hydrodynamic models in describing experimental data in high energy nuclear collisions [1-7] as well as cold atom systems [8-11]. In these systems the initial state is far from local thermal equilibrium, and it is not fully understood how hydrodynamic behavior emerges. The search for a kinetic framework that describes far-fromequilibrium plasmas has been one of the most important research subjects in high energy nuclear collisions and condensed matter physics [12-14].

Published by the American Physical Society under the terms of the Creative Commons Attribution 4.0 International license. Further distribution of this work must maintain attribution to the author(s) and the published article's title, journal citation, and DOI. Funded by SCOAP .
An important development in nonequilibrium dynamics was the discovery of emergent hydrodynamic behavior in far-from-equilibrium conditions which can be understood in terms of the mathematical theory of resurgence [15]. In this work the authors consider an extended hydrodynamic model, the Israel-Stewart equation [16], and apply it to a strongly coupled plasma undergoing Bjorken expansion. Subsequently, similar findings were obtained in many other transport models. These results show a deep connection between nonlinear relaxation toward hydrodynamic behavior, also known as "hydrodynamization," and transasymptotics and transseries [17-26].

Since then a very interesting and rich physical picture has emerged: The nonlinear relaxation process toward hydrodynamic behavior, also known as hydrodynamization, is driven by the decay of nonhydrodynamic degrees of freedom. Once the nonhydrodynamic modes have died out the system enters into the hydrodynamic attractor which is entirely determined by the standard asymptotic gradient expansion. This new insight might be able to explain why hydrodynamic models work very well when applied in extreme experimental scenarios such as ultrarelativistic heavy ion collisions $[27,28]$. 
Another interesting development in the understanding of far-from-equilibrium attractors in relativistic nonequilibrium dynamics is a phase space analysis using the language of nonautonomous dynamical systems ${ }^{1}$ [29-32]. This particular point of view allows us to characterize in simple terms the behavior of the solutions, described as flows, either at early or at late times. For instance, a global and local phase space flow analysis led to the conclusion that a large class of kinetic models undergoing Bjorken expansion [33] hydrodynamize in the long-time limit $[21,31,32,34]$, whereas systems undergoing Gubser flow [35,36] never do [29,32]. Moreover, the flow structure in phase space together with the symmetries of the dynamical system constrains the asymptotic behavior of the solutions of the ordinary differential equations (ODEs). For example, for weakly coupled boost-invariant systems it was demonstrated [31] that the solutions of the moments' equations admit power-law series expansions at early times, while at late times linear perturbations of the moments decay exponentially $[21,30,31,37]$. This is due to the nature of the early- and late-time attractors.

Attractors are understood as regions in phase space where flows accumulate either in the long- or short-time limit. However, in nonautonomous dynamical systems the past and future of the evolution are not the same since time translation invariance is explicitly broken. Each flow solution $\phi \equiv \phi\left(\phi_{0} ; t, t_{0}\right)$ is written in terms of its initial value $\phi_{0}$ and its initial and final times, $t_{0}$ and $t$, respectively. In this context, it is important to differentiate the backward and forward asymptotic regions of the dynamical system. We refer to a forward attractor as an asymptotic limit of the flows at which solutions converge when $t \rightarrow \infty$ while the initial time $t_{0}$ is fixed. In contrast, the pullback attractor is defined in the limit $t_{0} \rightarrow 0$ while keeping $t$ fixed. It is important to emphasize that for nonautonomous dynamical systems both limits do not commute [38,39].

Most previous work on the convergence properties of kinetic equations in relativistic transport theory was based on very simple collision terms, e.g., the relaxation time approximation (RTA). In this work we study the resurgent asymptotic properties of the Boltzmann equation for a boost-invariant Yang-Mills plasma governed by the weak coupling collision term in the small-angle approximation. In this limit the Boltzmann equation can be written as a nonlinear Fokker-Planck equation.

\footnotetext{
${ }^{1}$ A nonautonomous dynamical system corresponds to a general set of ODEs for the vector a of the generic form

$$
\frac{d \mathbf{a}}{d \lambda}=\mathcal{H}(\mathbf{a}, \lambda)
$$

where $\mathcal{H}$ is a function that depends explicitly on both a and $\lambda$. When the affine parameter $\lambda$ does not appear explicitly in the rhs of the previous expression it is said that the system is autonomous.
}

The Fokker-Planck equation (FPE) for gluons is of great interest since it captures essential aspects of the early-time dynamics of QCD matter produced in ultrarelativistic heavy ion collisions $[40,41]$. Due to the highly nonlinear structure of the collision kernel in the small-angle approximation, the FPE has been solved mostly by numerical means [42-48]. Very few analytical solutions for rapidly expanding systems are known in the literature [49]. One of the main achieved goals in this work is to fill this gap.

Following Grad's method [50] we map the mathematical problem of solving the FPE onto finding solutions to the kinetic equations for the Legendre moments $c_{l}$ [29-32]. The moment method turns out to be not only quickly convergent from a numerical point of view (see Appendix G of Ref. [31]), but it also provides a unique approach to understand nontrivial aspects of hydrodynamization. New solutions to the equations of motion of Legendre moments are derived by employing methods developed in the context of stability analysis of nonautonomous dynamical systems $[38,39]$, as well as techniques from superasymptotic and hyperasymptotic analysis [51-54]. These tools allow us to analyze the hydrodynamization process in two distinct regimes characterized by the size of dissipative corrections: $K n \gg 1$ (UV, early time) and $K n \ll 1$ (IR, late time).

The solutions of the kinetic equations for the Legendre moments enable us to extend the definition of transport coefficients to the far-from-equilibrium regime. Within our approach transport coefficients depend on the deformation history of the fluid, i.e., its rheology, and their values change as a function of the Knudsen number. Such dependence of the transport coefficients on the typical gradient size is a salient property of non-Newtonian fluids. Our findings provide further evidence for the connection between hydrodynamization and the transient rheological behavior of the plasma [30,31].

An interesting aspect of our study is the fact that the evolution of the transport coefficients is determined by a $\mathrm{RG}$ flow equation where the role of the RG scale is played by the Knudsen number. Our work draws inspiration from recent arguments that any RG flow can be viewed as a dynamical system [55,56]. We show that, conversely, certain dynamical systems can be understood as RG flows, provided the existence of the slow invariant manifold. This idea was first explored in the case of boost-invariant plasmas governed by a Boltzmann equation in the RTA approximation [30-32].

Finally, our study naturally explains the origin of the UV power series expansion considered first in [31] and later studied in [26,57-59]. The power series emerges by analyzing the stability behavior of solutions close to the UV fixed point. It is shown that the finite radius of convergence of the UV power series expansion depends on the value of the shear viscosity over entropy ratio $\eta / s$. We also outline the intriguing universal properties of the FPE related to the pullback and forward attractors and 
compare them with other kinetic models such as the RTA and the full leading-order Arnold-Moore-Yaffe (AMY) kinetic theory [60].

The outline of this work is as follows. In the next section we review the basic ideas behind the Fokker-Planck (FP) equation and introduce the expansion of the distribution function in terms of its moments whose evolution equations are derived. For pedagogical purposes we study extensively a truncation of this dynamical system in Sec. III where we present in detail the transasymptotic techniques studied in this article. In Sec. IV we demonstrate that the general solutions of the Fokker-Planck equation are written in terms of multiparameter transseries after resumming the fluctuations around the UV and IR regimes, respectively. We analyze the universal properties of nonequilibrium YangMills attractors of different theories in Sec. V. We conclude by giving final remarks. The technical details of our work are presented in the Appendixes.

\section{YANG-MILLS TRANSPORT EQUATION IN THE SMALL-ANGLE APPROXIMATION}

We consider an interacting gluon plasma described by the Boltzmann equation in the small-angle approximation [61]. We focus our discussion on the case of an expanding system with longitudinal boost invariance [33]. The dynamics is invariant under the $I S O(2) \otimes S O(1,1) \otimes$ $Z_{2}$ symmetry group. The symmetry becomes manifest in Milne coordinates $x^{\mu}=(\tau, x, y, \varsigma)$, where $\tau=\sqrt{t^{2}-z^{2}}$ is the longitudinal proper time, and $\varsigma=\tanh ^{-1}(z / t)$ is the spatial rapidity. In this coordinate system the metric is simply $g_{\mu \nu}=\left(-1,1,1, \tau^{2}\right)$. In Milne coordinates the Fokker-Planck equation for the one-particle distribution $f\left(\tau, p_{T}, p_{\varsigma} / \tau\right) \equiv f_{p}$ is $[40,46,47]$

$$
\partial_{\tau} f_{\boldsymbol{p}}=\mathcal{C}\left[f_{\boldsymbol{p}}\right]
$$

The collision kernel $\mathcal{C}\left[f_{p}(\tau)\right]$ in the small-angle approximation takes the form $[40,46]$

$$
\mathcal{C}\left[f_{p}\right]=\lambda_{Y M}^{2} l_{C b} \nabla_{\mathbf{p}} \cdot\left[\mathcal{J} \nabla_{\mathbf{p}} f_{\mathbf{p}}+\mathcal{K} \frac{\mathbf{p}}{p} f_{\mathbf{p}}\left(1+f_{\mathbf{p}}\right)\right],
$$

where we introduce the 't Hooft coupling $\lambda_{Y M}=$ $g^{2} N_{c} \equiv 4 \pi \alpha_{s} N_{c}$. The integrals $\mathcal{J}$ and $\mathcal{K}$ are given by

$$
\begin{gathered}
\mathcal{J}=\mathcal{J}(\tau)=\int \frac{d^{3} p}{(2 \pi)^{3}} f_{\mathbf{p}}\left[1+f_{\mathbf{p}}\right], \\
\mathcal{K}=\mathcal{K}(\tau)=2 \int \frac{d^{3} p}{(2 \pi)^{3}} \frac{f_{\mathbf{p}}}{p} .
\end{gathered}
$$

The Coulomb logarithm $l_{C b}$ in the rhs of Eq. (2) is a divergent integral of the form

$$
l_{C b}=\int_{p_{\min }}^{p_{\max }} \frac{d p}{p}=\log \left(\frac{p_{\max }}{p_{\min }}\right) .
$$

The IR momentum divergence originates from $2 \rightarrow 2$ scattering with small momentum transfer. In QCD these divergences are regularized by static and dynamic screening. The corresponding mass scale is on the order of the Debye mass. Near equilibrium, and for particles obeying Bose-Einstein statistics, we have [62]

$$
m_{D}^{2}=4 N_{c} g^{2} \int \frac{d^{3} p}{(2 \pi)^{3}} \frac{f_{\mathbf{p}}^{\text {eq. }}}{p}=2 \frac{\zeta(2) \Gamma(2)}{\pi^{2}} \lambda_{Y M} T^{2},
$$

where $\zeta(n)$ and $\Gamma(n)$ are the Riemann and Gamma functions, respectively. The UV momentum cutoff is taken to be the mean $p_{T}^{2}$, which close to equilibrium is

$$
\left\langle p_{T}^{2}\right\rangle=\frac{\int d^{3} p p_{T}^{2} f_{\mathbf{p}}^{\text {eq. }}}{\int d^{3} p f_{\mathbf{p}}^{\text {eq. }}}=2 \frac{\zeta(5) \Gamma(5)}{\zeta(3) \Gamma(3)} T^{2} .
$$

As a result, near equilibrium the Coulomb logarithm (4) is approximately given by

$l_{C b}=\int_{m_{D}}^{\sqrt{\left\langle p_{T}^{2}\right\rangle}} \frac{d p}{p}=\log \left(\frac{\sqrt{\left\langle p_{T}^{2}\right\rangle}}{m_{D}}\right) \sim \frac{1}{2} \log \left(\frac{\mathcal{A}}{\lambda_{Y M}}\right)$,

with $\mathcal{A}=72 \zeta(5) / \zeta(3) \approx 62.1$. This estimate gives a Coulomb logarithm which is a constant, independent of the energy density of the medium, but logarithmically dependent on the coupling constant. This approximation has been used in many previous studies $[45,46,59,63]$. Replacing $f_{\mathbf{p}}^{\text {eq. }}$ with the general nonequilibrium $f_{\mathbf{p}}$ in (5) and (6) and evaluating the integrals numerically at each time step allowed the authors of Refs. [42,48] to account for the time dependence of the UV and IR momentum cutoffs.

The approximation of a constant Coulomb logarithm gives the correct dependence of the shear viscosity on the coupling constant in the near-equilibrium, weak coupling limit. However, the numerical prefactor does not agree with calculations based on the full hard thermal loop (HTL) result $[64,65]$. This issue was addressed in [66], where the authors propose a simple regulator that reproduces the leading-order HTL result for drag and momentum diffusion in soft $2 \rightarrow 2$ scattering. This is a useful prescription, but it does not affect the late-time emergent hydrodynamic behavior. We shall comeback to this issue in Sec. V.

\section{A. Ansatz for the distribution function}

One of the most widely used methods to solve the Boltzmann equation was developed decades ago by Grad [50]. In this approach the problem of solving the Boltzmann equation is converted into a set of nonlinear partial differential equations (PDEs) for moments of the one-particle distribution function. This approach is very 
useful when analyzing the resurgence properties of the nonlinear ODEs for the moments [29-32].

In this work we will consider the following ansatz for the distribution function in a boost-invariant system [30-32]:

$$
f_{p}=f_{p}^{\mathrm{eq}} \sum_{\ell=0}^{+\infty} c_{\ell}(\tau) P_{2 \ell}\left(\cos \theta_{p}\right),
$$

where $p^{\tau}=\sqrt{p_{x}^{2}+p_{y}^{2}+\left(p_{\zeta} / \tau\right)^{2}}, \cos \theta_{p}=p_{\zeta} /\left(\tau p^{\tau}\right)$, and $P_{2 l}$ are the Legendre polynomials. In Eq. (8) the equilibrium distribution function $f_{p}^{\mathrm{eq}}$ is

$$
f_{\boldsymbol{p}}^{\mathrm{eq}}=\nu_{\mathrm{eff}} \frac{1}{e^{p^{\tau} / T(\tau)}-1}
$$

where $T$ is the temperature of the system which is defined below via the Landau matching condition and $\nu_{\text {eff }}$ are the effective degrees of freedom which for simplicity we set to be $\nu_{\text {eff }} \equiv 1$. We implicitly assume that $f_{p}$ is independent of spin and color. The ansatz (8) is consistent with the restrictions imposed by $\operatorname{ISO}(2) \otimes \mathrm{SO}(1,1) \otimes Z_{2}$. In particular, the distribution function is invariant under the action of the Killing vectors $\phi_{i}$ of this symmetry group, i.e., $\partial f_{p} / \partial \phi_{i}=0$ [30-32]. For simplicity we fixed $\mu \equiv 0$ in the equilibrium distribution function (9). Furthermore, the ansatz (8) does not carry information about the nonlinear relaxation of the transient high energy tails of the distribution function which have been studied previously within the moment method [30-32,46,47,67-70]. A more general ansatz which encodes some of the information on the high energy tails was discussed recently in Ref. [45].

The Legendre moments $c_{\ell}$ are directly determined by Eq. (8),

$$
c_{\ell}=\frac{30(4 l+1)}{\pi^{2} T^{4}}\left\langle(-u \cdot p)^{2} P_{2 \ell}\left(\cos \theta_{p}\right)\right\rangle,
$$

where we denote $\langle\cdots\rangle_{X} \equiv \int_{p} \cdots f_{p}^{X}$ and the momentum measure is $\int_{p} \equiv \int d^{2} p_{T} d p_{\varsigma} /\left[(2 \pi)^{3} \tau p^{\tau}\right]$. If the system reaches the thermal equilibrium state, the moments $c_{l}^{\text {eq. }}=\delta_{l 0}$.

For the Bjorken flow the energy-momentum tensor $T^{\mu \nu}=\left\langle p^{\mu} p^{\nu}\right\rangle$ is given by $[30-32,71,72]$

$$
T^{\mu \nu}=\epsilon u^{\mu} u^{\nu}+\mathcal{P}_{L} l^{\mu} l^{\nu}+\mathcal{P}_{T} \Xi^{\mu \nu},
$$

where we denote the timelike vector identified with the fluid velocity $u^{\mu}=(1,0,0,0)$ (with $u^{\mu} u_{\mu}=-1$ ), the spacelike normal vector pointing along the $\varsigma$ direction is $l^{\mu}=(0,0,0,1)\left(\right.$ with $\left.l^{\mu} l_{\mu}=1\right)$ and the projection operator $\Xi^{\mu \nu}=g^{\mu \nu}+u^{\mu} u^{\nu}+l^{\mu} l^{\nu}$ which is orthogonal to both $u^{\mu}$ and $l^{\mu}$, respectively. The energy density $\epsilon$, longitudinal and transverse pressures, $\mathcal{P}_{L}$ and $\mathcal{P}_{T}$, respectively, are written in terms of the angular moments as follows:

$$
\begin{gathered}
\epsilon=\left\langle(-u \cdot p)^{2}\right\rangle=\frac{\pi^{2}}{30} c_{0} T^{4}, \\
\mathcal{P}_{T}=\left\langle\frac{1}{2} \Xi^{\mu \nu} p_{\mu} p_{\nu}\right\rangle=\epsilon\left(\frac{1}{3}-\frac{1}{15} c_{1}\right), \\
\mathcal{P}_{L}=\left\langle(l \cdot p)^{2}\right\rangle=\epsilon\left(\frac{1}{3}+\frac{2}{15} c_{1}\right) .
\end{gathered}
$$

It follows that $\epsilon=2 \mathcal{P}_{T}+\mathcal{P}_{L}$ as expected. The Landau matching condition for the energy density $\epsilon=\epsilon_{\text {eq. }} \equiv\langle(-u$. $\left.p)^{2}\right\rangle_{\text {eq. }}$ implies $c_{0} \equiv 1$. For the Bjorken case the normalized pressure anisotropy is the ratio of the independent shear viscous tensor over the energy is written in terms of the Legendre moment $c_{1}$, i.e.,

$$
\Delta=\frac{\tau^{2} \pi^{\varsigma \varsigma}}{\epsilon}=\frac{2}{15} c_{1}
$$

Moreover, the non-negativity property of the longitudinal and transverse pressures $\mathcal{P}_{L(T)} \geq 0$ implies $-5 / 2 \leq c_{1} \leq 5$. This bounds the basin of attraction from below and above and is satisfied in general only by the exact kinetic solution to the FP equation (2). Nonetheless, it is known that any perturbative approach which aims to find an approximate solution to the Boltzmann equation does not necessarily obey this constraint [73].

\section{B. Evolution equation of the Legendre moments evolution}

We truncate the expansion in Legendre polynomials at order $L$ and write the Legendre moments as a vector $\mathbf{c}(\tau) \equiv\left(c_{1}(\tau), \ldots, c_{L}(\tau)\right)^{\top}$. Following the procedure outlined in [30-32] we find that the evolution equations for the temperature and the Legendre moments are given by the following coupled nonlinear ODEs:

$$
\begin{gathered}
\frac{d T}{d \tau}=-\frac{T}{3 \tau}\left(1+\frac{c_{1}}{10}\right), \\
\frac{d \mathbf{c}}{d \tau}=\mathbf{F}(T, \mathbf{c}, \tau),
\end{gathered}
$$

with

$$
\begin{aligned}
\mathbf{F}(T, \mathbf{c}, \tau)= & -\frac{2}{3}\left[\frac{1}{\tau}\{\mathfrak{X}(\mathbf{c}) \mathbf{c}+\boldsymbol{\Gamma}\}\right. \\
& +\{\Lambda+\mathfrak{Y}(\mathbf{c})+\mathcal{Z}(\mathbf{c})\} T \mathbf{c}]
\end{aligned}
$$

and

$$
\mathfrak{X}(\mathbf{c})=\overline{\mathfrak{X}}-\frac{1}{5} c_{1}(\tau) \mathbf{1}_{L},
$$




$$
\overline{\mathfrak{X}}=\frac{3}{2}\left(\begin{array}{ccccc}
\mathfrak{B}_{1} & \mathfrak{A}_{1} & & & \\
\mathfrak{S}_{2} & \mathfrak{B}_{2} & \mathfrak{A}_{2} & & \\
& \ddots & \ddots & \ddots & \\
& & \mathfrak{C}_{L-1} & \mathfrak{B}_{L-1} & \mathfrak{A}_{L-1} \\
& & & \mathfrak{C}_{L} & \mathfrak{B}_{L}
\end{array}\right) .
$$

$$
\begin{gathered}
\mathfrak{B}_{l}=\frac{2\left(14 \ell^{2}+7 \ell-2\right)}{(4 \ell-1)(4 \ell+3)}-\frac{4}{3}, \\
\mathfrak{\mathfrak { c }}_{\ell}=\frac{2 \ell(2 \ell-1)(2 \ell+2)}{(4 \ell-3)(4 \ell-1)} .
\end{gathered}
$$

The Lyapunov exponents and the vector $\Gamma$ are defined by

The matrix elements are defined by

$$
\begin{gathered}
\Lambda=\operatorname{diag} .(\lambda(1), \ldots, \lambda(L)), \\
\Gamma=\left(3 \mathfrak{\mho}_{1} / 2,0, \ldots, 0\right)^{\top}=(4,0, \ldots, 0)^{\top} .
\end{gathered}
$$

Finally, we have defined the matrices

$$
\begin{gathered}
\mathfrak{Y}(\mathbf{c})=\hat{\kappa}\left(\begin{array}{ccccc}
\sum_{m=1}^{2} \Omega_{1 m 1} c_{m}(\tau) & \cdots & \sum_{m=n-1}^{1+n} \Omega_{1 m n} c_{m}(\tau) & \cdots & \sum_{m=L-1}^{1+L} \Omega_{1 m L} c_{m}(\tau) \\
\vdots & \ddots & \vdots & \ddots & \vdots \\
\sum_{m=\ell-1}^{\ell+1} \Omega_{\ell m 1} c_{m}(\tau) & \cdots & \sum_{m=\operatorname{Max}[\ell \ell-n \mid, 1]}^{\ell+n} \Omega_{\ell m n} c_{m}(\tau) & \cdots & \sum_{m=L-\ell}^{\ell+L} \Omega_{\ell m L} c_{m}(\tau) \\
\vdots & \ddots & \vdots & \ddots & \vdots \\
\sum_{m=L-1}^{L+1} \Omega_{L m 1} c_{m}(\tau) & \cdots & \sum_{m=L-n}^{L+n} \Omega_{L m n} c_{m}(\tau) & \cdots & \sum_{m=1}^{2 L} \Omega_{L m L} c_{m}(\tau)
\end{array}\right), \\
\Omega_{\ell m n}=\frac{\alpha_{m-n+\ell} \alpha_{n+m-\ell} \alpha_{n-m+\ell}}{\alpha_{n+m+\ell}} \cdot \frac{4 \ell+1}{2(n+m+\ell)+1}, \\
3(\mathbf{c})=\hat{\kappa} \sum_{n=1}^{+\infty} \frac{(2 \ell-1)(\ell+1)}{3(4 n+1)} c_{n}(\tau)^{2} \mathbf{1}_{L} .
\end{gathered}
$$

In the previous expressions the parameters $\kappa, \theta_{0}$ and $\hat{\kappa}$ are given by, respectively,

$$
\begin{aligned}
& \kappa=\pi^{2} / 3-2 \zeta(3), \quad \theta_{0}^{-1}=\frac{5}{8 \pi^{5}} \lambda_{Y M}^{2} l_{C b}, \\
& \hat{\kappa}=\frac{3 \kappa}{2 \theta_{0}}, \quad \lambda(l)=\frac{3}{2 \theta_{0}}\left(\kappa+\frac{l(2 l+1)}{3} \pi^{2}\right) .
\end{aligned}
$$

The derivation of the equations of motion (14) is presented in Appendix A. The set of ODEs (14) constitutes a nonlinear nonautonomous dynamical system [38,39] due to the explicit dependence on the proper time $\tau$ in the rhs of these equations. The strength of the effect of the collisions enters in the ODEs via the parameter $\theta_{0}$ (20); we thus will vary this parameter instead of the 't Hooft coupling $\lambda_{Y M}$ when showing the numerical results. The nonlinear nature of the FP equation (1) is manifest in the mode-mode couplings among different moments and in the temperature appearing in the rhs of Eq. (14). Finally, the time evolution of the energy-momentum tensor (11) can be fully reconstructed from the solutions of the temperature $T$ and the full set of Legendre moments $c_{l}$.

The equations of motion of the Legendre moments (10) for the conformal Boltzmann equation within the RTA are given by (see [30,31])

$$
\begin{aligned}
\frac{d \mathbf{c}}{d \tau} & =\mathbf{F}_{R T A}(T, \mathbf{c}, \tau), \\
\mathbf{F}_{R T A}(T, \mathbf{c}, \tau) & =-\frac{2}{3}\left[\frac{1}{\tau}\{\mathfrak{X}(\mathbf{c}) \mathbf{c}+\boldsymbol{\Gamma}\}+\Lambda_{R T A} T \mathbf{c}\right], \\
\Lambda_{R T A} & =\frac{3}{2 \Theta_{0}} \operatorname{diag} .(1,1, \ldots, 1) .
\end{aligned}
$$

Here $\Theta_{0}$ is a proportionality constant between the relaxation time and the shear viscosity over entropy ratio. This constant can be determined from relativistic kinetic theory methods which for the conformal RTA approximation gives us $\tau_{r}=\frac{5}{T} \frac{\eta}{s}[31,74-85]$, i.e., $\Theta_{0} \equiv 5 \eta / s$.

At linear order the form of the evolution equations for the Legendre moments for the RTA and FP, Eqs. (21) and (14), respectively, is very similar. At this order the main 
difference between the two models lies in the specific relaxation scale of the associated mode $c_{l}$. For the RTA approximation all modes decay with the same relaxation scale $\tau_{r}$ while in the Fokker-Planck case each $c_{l}$ has a characteristic relaxation scale $\lambda(l)^{-1}$ which increases as a function of the $l$ [see Eq. (20)]. Therefore for the FPE there is a clear hierarchy of fast and slow modes. ${ }^{2}$

The nonlinearities of the FPE are encoded in the mode coupling between moments of different order and in the implicit dependence of the temperature on $c_{1}$. These effects drive the system away from equilibrium and delay the relaxation to the equilibrium state. For instance, for the RTA Boltzmann equation a new nonhydrodynamic mode survives in the long wavelength limit due to a nonlinear coupling to the shear viscous tensor [31]. This result contradicts the common assumption of taking the typical relaxation timescale of a mode as a guide for constructing effective theories in the long-time limit.

\section{Dimensional reduction}

There is an important simplification of the nonlinear ODEs (14) which makes the asymptotic analysis simpler. It is possible to dimensionally reduce this dynamical system from $L+1 \rightarrow L$ by introducing the variable ${ }^{3} w=\tau T(\tau) \in \mathbb{R}^{+}$. Since $w \sim K n^{-1}$ [29-32], the variable $w$ encodes the strength of the dissipative corrections. This fact will be important for reinterpreting the dynamical system of ODEs as RG flows in Sec. III B 1. In terms of $w$ Eqs. (14) can be written as

$$
\begin{gathered}
T(w)=\mathcal{D}\left(w, w_{0}\right) T_{0}, \\
\mathcal{D}\left(w, w_{0}\right)=\exp \left[-\frac{1}{2} \int_{w_{0}}^{w} \frac{d w^{\prime}}{w^{\prime}} \frac{\left(1+\frac{c_{1}\left(w^{\prime}\right)}{10}\right)}{\left(1-\frac{c_{1}\left(w^{\prime}\right)}{20}\right)}\right], \\
\frac{d \mathbf{c}}{d w}=\mathbf{F}(\mathbf{c}, w), \\
\mathbf{F}(\mathbf{c}, w)=-\frac{1}{1-\frac{1}{20} c_{1}(w)}\left[\frac{1}{w}\{\mathfrak{X}(\mathbf{c}) \mathbf{c}(w)+\mathbf{\Gamma}\}\right. \\
+\{\Lambda+\mathfrak{Y}(\mathbf{c})+\mathfrak{Z}(\mathbf{c})\} \mathbf{c}(w)]
\end{gathered}
$$

The dynamics of this dimensionally reduced system of ODEs depends only on the Legendre moments $c_{l}{ }^{4}$ and does

\footnotetext{
${ }^{2}$ For hard spheres and within the kinetic theory framework similar results have been found in the past. In those models a hierarchy of scales between the fast and soft modes also emerges [68-70].

${ }^{3}$ The resurgence analysis can also be studied in terms of the original variable $\tau$ [31]. See the general Fokker-Planck case in Appendix $\mathrm{C}$ and the RTA Boltzmann case is extensively discussed in Appendixes A-D in Ref. [31].

${ }^{4}$ The index $i$ of vectors and matrices runs from $i=1,2, \ldots, L$. If one performs a truncation of the nonautonomous dynamical system of Eq. (22) it is understood that $c_{l} \equiv 0$ for $l>L$.
}

not involve the temperature variable explicitly. It is important to emphasize that the dimensionally reduced dynamical system does not preserve the topological properties of the original nonlinear ODEs (14). For instance, a new coordinate singularity emerges when $c_{1}=20$ [see the denominator in the integrand of the damping function $D\left(w, w_{0}\right)$ in Eq. (22a)] which does not exist in the $\tau$ variable. Furthermore, in the $w$ variable, one can find a set of bounded solutions at $w_{0} \rightarrow 0$ for a fixed $w$, the so-called pullback attractor [31,32]; whereas the singularity at $\tau=0$ forbids the existence of any bounded solutions in the UV. This is of course expected on many grounds but a simple explanation is that the system undergoes a topological change under $\tau \rightarrow w$ that lifts the $\tau=0$ singularity at the expense of introducing a new singularity at $c_{1}=20[31,32]$.

\section{TRANSASYMPTOTIC ANALYSIS: THE TRUNCATED $L=1$ CASE}

Before presenting the transseries solutions for the nonlinear ODEs (22) we first illustrate our techniques by considering the case where the system is truncated to a single nonhydrodynamic degree of freedom. Physically, this degree of freedom corresponds to the viscous shear tensor. This warm-up exercise illustrates the main aspects of the general asymptotic analysis to be discussed in Sec. IV.

Let us assume that the moments $c_{l} \equiv 0$ for $l>1$. In terms of the variable $w$, the truncated $l=1$ case read as

$$
\begin{gathered}
\frac{d c_{1}}{d w}=F_{1}\left(w, c_{1}\right), \\
F_{1}\left(w, c_{1}\right)=-\frac{1}{\left(1-\frac{1}{20} c_{1}\right)}\left[\frac{1}{w}\left(4+\frac{5}{7} c_{1}-\frac{1}{5} c_{1}^{2}\right)\right. \\
\left.+\lambda(1) c_{1}+\frac{2 \hat{\kappa}}{7} c_{1}^{2}+\frac{2 \hat{\kappa}}{15} c_{1}^{3}\right] .
\end{gathered}
$$

Before discussing the transasymptotic analysis we would like to comment on the existence of the pullback and forward attractors of the truncated ODE (23a). The extension to the full set of nonlinear ODEs (22) is similar but more difficult to visualize [31]. For now, we note that we can identify attractors by inspecting the flow diagram of the ODE (23a). We will characterize the attractors more fully below. In the top panel of Fig. 1 we observe that in the IR regime all flows converge asymptotically toward the value $c_{1} \rightarrow 0$ when $w \gg 1$ regardless of their origin. This shows that there is only one IR fixed point associated with the forward attractor.

In the UV limit, on the other hand, there are two fixed points (black dots in Fig. 1) which are located along the $c_{1}$ axis in the small $w_{0} \rightarrow 0$ limit ( $w_{0}$ is the initial value of $w$ ). The UV behavior of the flow lines in each fixed point is rather different. For the UV fixed point located in the 

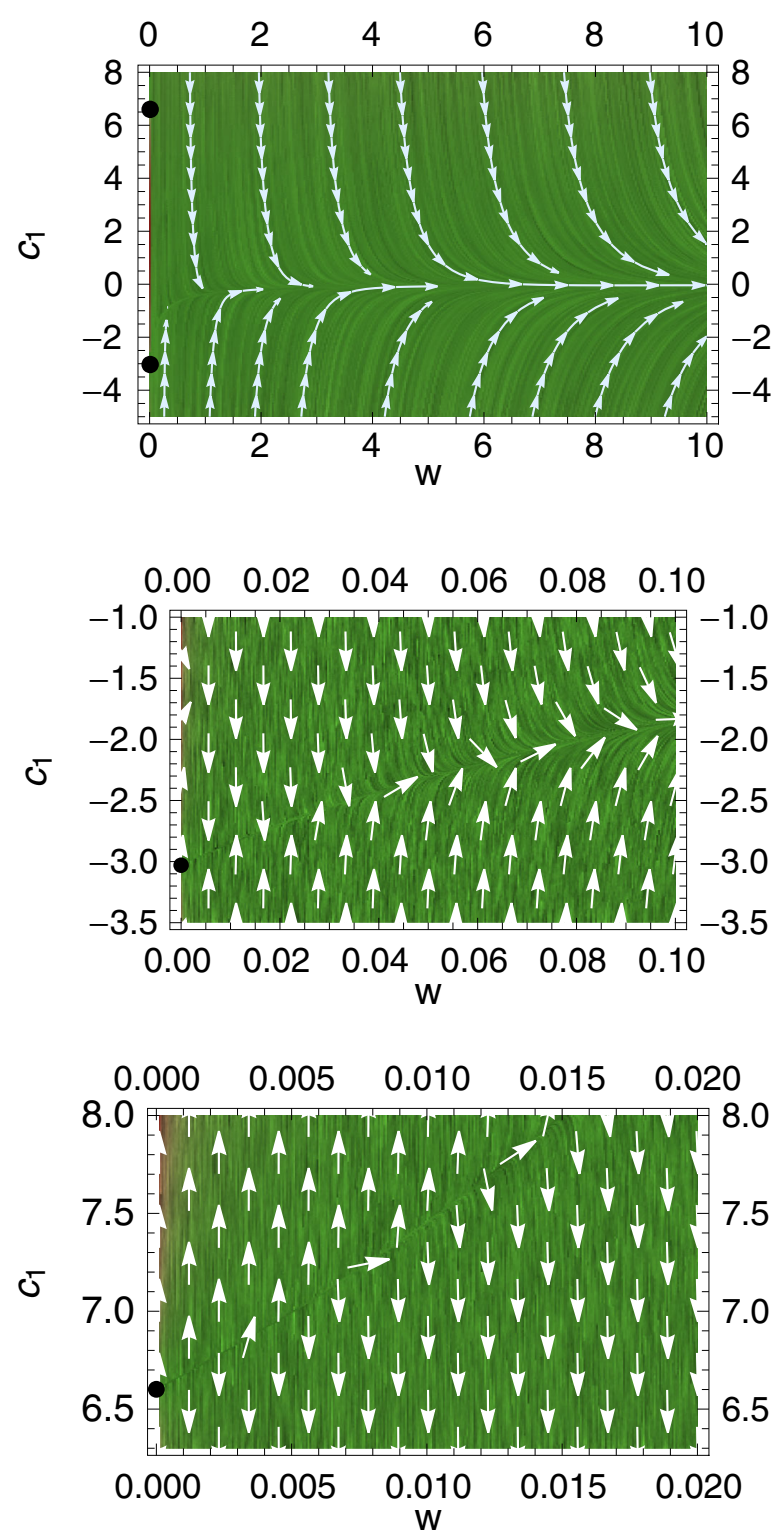

FIG. 1. Flow diagram of the differential equation for the Legendre moment (23a) in the $\left(c_{1}, w\right)$ space. Top, center, and bottom panels show the flows close to the IR and two UV fixed points, respectively. The black dots showed in each panel correspond to the two UV fixed points of the ODE (23a).

positive $c_{1}$ region one observes that the flow lines emerge out of it in all the directions, and thus this fixed point is a source. In the negative $c_{1}$ region, we observe that near the UV fixed point the flow lines coming from above or below repel each other so the fixed point is a saddle. However, although the flow lines repel each other, they merge quickly in the vicinity of this fixed point. Thus, the UV fixed point located in the negative $c_{1}$ region is identified as the pullback attractor.

We will see that the behavior of the transseries solution near these fixed points is rather different. We note that the behavior of the flows in the $\tau$ variable differs from the one in $w$. In the former case the temperature is a dynamical variable. It diverges in the limit $\tau \rightarrow 0$ due to the presence of the singularity at $\tau=0$; therefore there is no meaningful definition of a pullback attractor in the $\tau$ variable (see also $[31,32])$.

\section{A. Transseries solution in the IR limit}

The leading-order term of each Legendre moment in Eqs. (22) is $c_{l}=\mathcal{O}\left(w^{-l}\right) \forall l>0$ in the IR regime [31]. Hence, Eq. (23a) admits the following asymptotic expansion for $c_{1}$ :

$$
c_{1}=\sum_{k=0}^{\infty} u_{1, k}^{(0)} w^{-k}
$$

The coefficients $u_{1, k}^{(0)}$ are obtained simply by inserting the IR expansion into the original ODE (23a). For instance, the first three coefficients are given by

$$
\begin{aligned}
& u_{1,0}^{(0)}=0, \quad u_{1,1}^{(0)}=-\frac{4}{\lambda(1)}, \\
& u_{1,2}^{(0)}=-\frac{8}{7 \lambda(1)^{2}}\left(1+4 \frac{\hat{\kappa}}{\lambda(1)}\right) .
\end{aligned}
$$

Here the reality condition of $c_{1}$ was implicitly taken into account. The hydrodynamic gradient expansion of the shear viscous tensor [86] provides the following expression for $c_{1}[30,31]$ :

$$
c_{1}=-\frac{40}{3} \frac{1}{w} \frac{\eta}{s}-\frac{80}{9} \frac{1}{w^{2}} \frac{T\left(\eta \tau_{\pi}-\lambda_{1}\right)}{s} \cdots,
$$

where we use Eq. (13). Comparing the previous expression and Eqs. (25) one concludes

$$
\begin{aligned}
\frac{\eta}{s} & =\frac{3}{10} \frac{1}{\lambda(1)}, \\
\frac{T\left(\eta \tau_{\pi}-\lambda_{1}\right)}{s} & =\frac{9}{70 \lambda(1)^{2}}\left(1+4 \frac{\hat{\kappa}}{\lambda(1)}\right),
\end{aligned}
$$

with $\lambda(1)$ and $\hat{\kappa}$ given in Eqs. (20). We will see below that $\lambda(1)$ is related to the rate at which fluctuations decay close to the IR fixed point, i.e., the Lyapunov exponent. The coefficients $u_{1, k}^{(0)}$ that enter the IR expansion (24) are understood as transport coefficients in the IR limit. Furthermore, the set of the identities (27) turn out to be of importance when generalizing the concept of a transport coefficient to the far-from-equilibrium regime [30,31] as we shall discuss in Sec. III B.

It is known that the IR expansion (24) is divergent since its coefficients grow factorially [15]. These expansions are merely formal expressions and emerge as asymptotic 
solutions of a certain class of differential equations [52]. In order to see this let us simply perform a linear perturbation around the perturbative expansion (24). Thus, by shifting $c_{1} \rightarrow \bar{c}_{1}+\delta c_{1}$ with $\bar{c}_{1}=\sum_{k=0}^{\infty} g_{k} w^{-k}$ while keeping terms up to $\mathcal{O}\left(w^{-1}\right)$ one obtains the following linearized differential equation for the perturbation $\delta c_{1}$ :

$$
\begin{aligned}
\frac{d \delta c_{1}}{d w} & =\left.\frac{\partial F_{1}}{\partial c_{1}}\right|_{c_{1}=\bar{c}_{1}} \delta c_{1}, \\
& \approx-\left(\lambda(1)+\frac{1}{w}\left(\frac{18}{35}-\frac{16}{7} \frac{\hat{\kappa}}{\lambda(1)}\right)\right) \delta c_{1},
\end{aligned}
$$

whose solution reads

$$
\begin{aligned}
\delta c_{1}(w) & =\sigma_{1} e^{-S_{1} w^{-b_{1}}}, \\
S_{1} & :=\lambda(1), \quad b_{1}:=\frac{18}{35}-\frac{16}{7} \frac{\hat{\kappa}}{\lambda(1)},
\end{aligned}
$$

where $\sigma_{1}$ is the integrating constant, $S_{1}$ is recognized as the Lyapunov exponent and $b_{1}$ is the anomalous dimension. The presence of the exponential terms is an indication that in order to capture the transient behavior of the solutions it is needed to go beyond the perturbative IR expansion (24).

The leading exponential term (29) is the first type of a large set of exponentials terms that appear when summing over the fluctuations around the IR fixed point. In order to include systematically these type of terms we follow Costin's prescription [54]. First, notice that in the IR limit the ODE (23a) takes the following asymptotic form:

$$
\begin{aligned}
\frac{d c_{1}}{d w} \approx & -\sum_{n=0}^{\infty}\left(\frac{c_{1}}{20}\right)^{n}\left[\frac{1}{w}\left(4+\frac{5}{7} c_{1}-\frac{1}{5} c_{1}^{2}\right)\right. \\
& \left.+\lambda(1) c_{1}+\frac{2 \hat{\kappa}}{7} c_{1}^{2}+\frac{2 \hat{\kappa}}{15} c_{1}^{3}\right], \\
\approx & -\left[\lambda(1) c_{1}+\frac{1}{w}\left(4+\frac{32}{35} c_{1}\right)\right]+R\left(c_{1}, w\right),
\end{aligned}
$$

where $R\left(c_{1}, w\right)$ is a nonlinear polynomial function of $c_{1}$ and $w$. The asymptotic limit of the differential equation (30) coincides with the prepared form of the generic class of differential equations studied by Costin [54]. Thus, given the regularity of the solutions at $w \rightarrow \infty$ as well as the nonvanishing value for the Lyapunov exponent $S_{1}$ (29), Eq. (23a) has an exact transseries solution [54]

$$
\begin{gathered}
c_{1}(w)=\sum_{k=0}^{\infty} \sum_{n=0}^{\infty} u_{1, k}^{(n)} w^{-k}\left[\sigma_{1} \zeta_{1}(w)\right]^{n}, \\
\zeta_{1}(w)=e^{-S_{1} w} w^{-b_{1}} .
\end{gathered}
$$

From the physical point of view, the transseries (31) describes deviations from thermal equilibrium due to exponentially damped modes multiplied by gradient terms. The exponential damped terms, usually called nonhydrodynamic modes, play a role analogous to instantons in quantum field theory and quantum mechanics. In order to determine the coefficients $u_{1, k}^{(n)}$ we simply equate (31) with Eq. (23a). As a result we get the following recursive relation:

$$
\begin{aligned}
& \left(-m S_{1}+\lambda(1)\right) u_{1, k}^{(m)}+\left(-m b_{1}-k+\frac{12}{7}\right) u_{1, k-1}^{(m)}+4 \delta_{m, 0} \delta_{k, 1} \\
& \quad-\frac{1}{20} \sum_{m_{1}, m_{2}=0}^{m_{1}+m_{2}=m} \sum_{k_{1}, k_{2}=0}^{k_{1}+k_{2}=k}\left[\left(-m_{1} S_{1}-\frac{40 \hat{\kappa}}{7}\right) u_{1, k_{1}}^{\left(m_{1}\right)}+\left(-m_{1} b_{1}-k_{1}+5\right) u_{1, k_{1}-1}^{\left(m_{1}\right)}\right] u_{1, k_{2}}^{\left(m_{2}\right)} \\
& \quad+\frac{2 \hat{\kappa}_{1}}{15} \sum_{m_{1}, m_{2}, m_{3}=0}^{m_{1}+m_{2}+m_{3}=m} \sum_{k_{1}, k_{2}, k_{3}=0}^{k_{2}+k_{3}=k} u_{1, k_{1}}^{\left(m_{1}\right)} u_{1, k_{2}}^{\left(m_{2}\right)} u_{1, k_{3}}^{\left(m_{3}\right)}=0
\end{aligned}
$$

where $u_{1, k}^{(m)}=0$ for $k<0$ and we take $u_{1,0}^{(1)}=1$ as the normalization of the integration constant $\sigma_{1}$. When $m=0$ and $k=0,1,2$ in the recursive relation (32) one reproduces Eqs. (25) as expected.

We conclude this section by presenting some numerical results. In Fig. 2 we show the Legendre mode $c_{1}$ as a function of $w$ for the exact numerical solution (red line), NavierStokes (NS, blue dotted line), NS + second order (green dashed line) and truncated transseries solutions where we added two (orange dashed line) and five (magenta dashed line) transmonomials to the first IR perturbative order. The initial condition for the numerical solution of the ODE (23a) was $c_{1}\left(w_{0}=0.03\right)=\{6.60119,0,-3.02976\}$ while fixing $\theta_{0}=1 .{ }^{5}$ Note that at each order of the resummed theory we have to numerically match the transseries parameter $\sigma$. Very few cases are known where one can determine $\sigma$ exactly [26]. In the present work we have determined $\sigma$ using a numerical least-square fit [31]. This leads to some uncertainty, which is not unexpected given the difficulty of matching the IR and UV data without performing an all-order resummation. The shaded yellow area in Fig. 2 shows the variation of $\sigma$ between each truncated transseries solution.

\footnotetext{
${ }^{5}$ The values for $c_{1}\left(w_{0}=0.03\right)=\{6.60119,-3.02976\}$ correspond to the UV fixed points, see Eq. (44).
} 

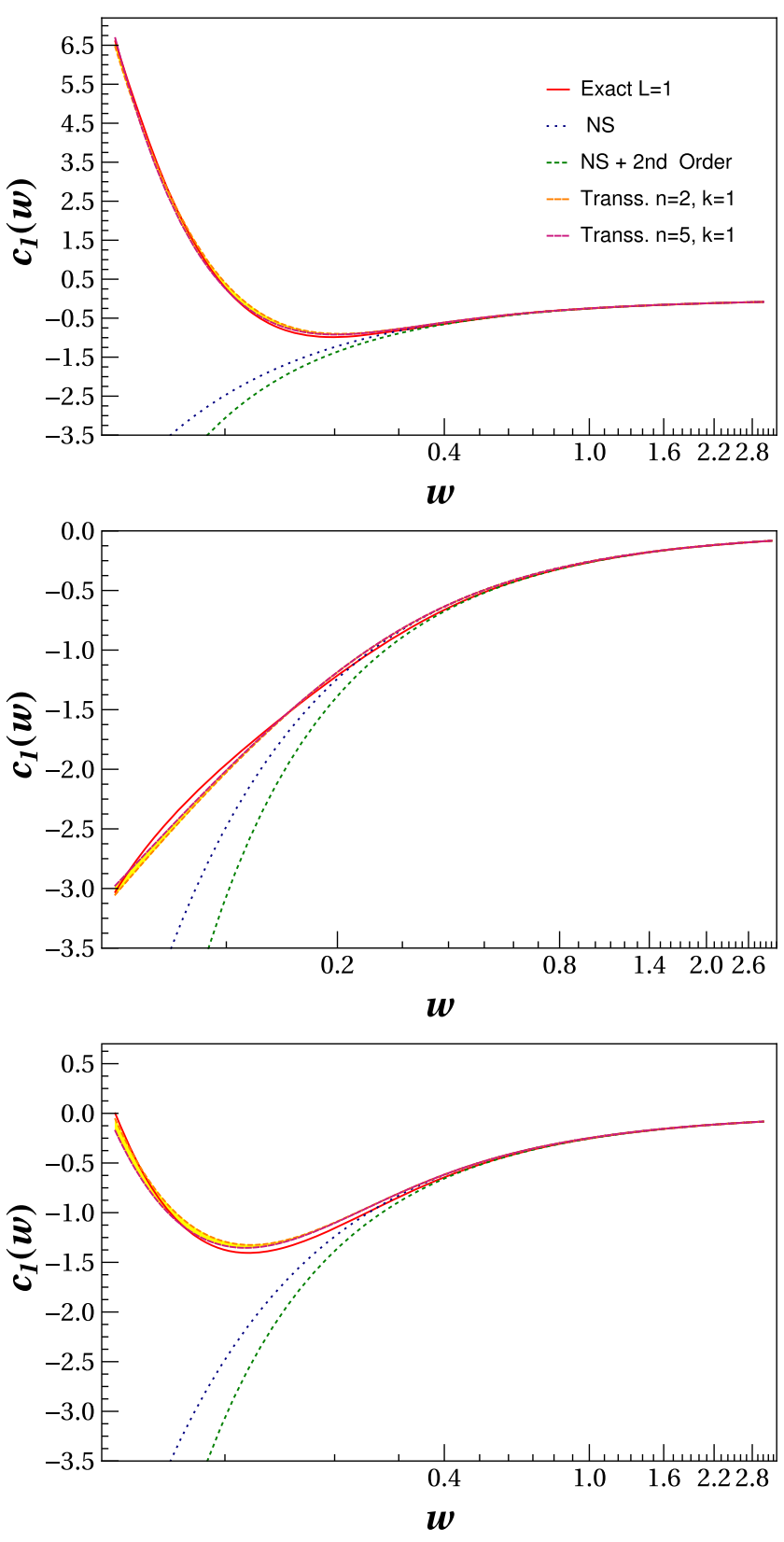

FIG. 2. Evolution of the nonhydrodynamic mode $c_{1}$ in terms of $w$ for the exact numerical result (red line), truncated transseries solutions by adding $n=2$ transmonomials to $k=1$ perturbative order (blue dotted line) and $n=15$ transmonomials to $k=3$ perturbative order (green dashed line) orders in the IR perturbative expansion, respectively, Navier-Stokes (orange dashed line) and NS + second order (magenta dashed line). See text for further details.

Figure 2 shows that the perturbative series at first and second orders does not match the numerical results for small values of $w$. On the other hand, the truncated transseries (31) where only a few transmonomials $(n=2,5$, respectively) were included is very close to the exact numerical result. We note that some care has to be taken in making such comparisons. Any truncated transseries will potentially exhibit large deviations from the exact numerical result in some range of $w$. This is due to the growth of the inherent error associated with any type of truncation scheme, see Appendix B. Any resummed perturbative series with a few transmonomials has a finite radius of convergence. The full transseries solution coincides with the exact theory only when summing over all the nonperturbative sectors of the perturbative series [54].

\section{B. Transasymptotic matching: $l=1$ case}

An interesting property of the IR transseries solution (31) emerges when rearranging the terms close to the IR fixed point. This procedure is known as "transasymptotic matching," and it is a well-known feature of transseries solutions for a general class of differential equations [53,54]. In the small $w$ limit there is a one-to-one competition between the exponentially decaying terms entering in the transseries and the inverse powers in $w$. However, close to the IR fixed point the instantonlike contributions are more suppressed than the leading IR perturbative terms. It is in this regime where one can indeed reshuffle and resum the small exponential terms such that

$$
\begin{aligned}
c_{1}(w)= & {\left[u_{1,0}^{(1)} \sigma_{1} \zeta_{1}(w)+u_{1,0}^{(2)}\left[\sigma_{1} \zeta_{1}(w)\right]^{2}+\cdots\right] } \\
& +\frac{1}{w}\left[u_{1,1}^{(0)}+u_{1,1}^{(1)} \sigma_{1} \zeta_{1}(w)+u_{1,1}^{(2)}\left[\sigma_{1} \zeta_{1}(w)\right]^{2}+\cdots\right] \\
& +\frac{1}{w^{2}}\left[u_{1,2}^{(0)}+u_{1,2}^{(1)} \sigma_{1} \zeta_{1}(w)+u_{1,2}^{(2)}\left[\sigma_{1} \zeta_{1}(w)\right]^{2}+\cdots\right]+\cdots \\
\equiv & \sum_{k=0}^{+\infty} G_{1, k}\left(\sigma_{1} \zeta_{1}(w)\right) w^{-k}
\end{aligned}
$$

with $\zeta_{1}(w)=e^{-S_{1} w^{-} w_{1}}$ and the transasymptotic functions $G_{k}\left(\sigma_{1} \zeta(w)\right)$ given by

$$
G_{1, k}\left(\sigma_{1} \zeta(w)\right)=\sum_{n=0}^{\infty} u_{1, k}^{(n)}\left[\sigma_{1} \zeta_{1}(w)\right]^{n} .
$$

The transasymptotic functions $G_{k}\left(\sigma_{1} \zeta(w)\right)$ effectively resum the full set of instantonlike contributions at a given order $k$ in perturbation theory. Interestingly this matching procedure is not only valid close to the IR but it extends up to the UV, so in this sense it is transasymptotic [52]. Each $G_{k}(\zeta(w))$ is an analytic, Borel summable and convergent functions even if one truncates at a given order the IR perturbative expansion Eq. (33). The full transseries solution (33) is not Borel summable due to the singularity of the original differential equation (23a). This singularity can be easily determined by taking the inverse Laplace transformation of the ODE (23a) (cf. Ref. [32]). The transasymptotic matching procedure coincides with the exact solution of the ODE only when summing over all the perturbative and nonperturbative sectors, i.e., $k, n \rightarrow \infty$ in the upper limit of the sums of Eq. (33) [52-54]. In general, 
a truncation of the perturbative expansion leads to a solution with a finite radius of convergence.

In the large $w$ limit the transasymptotic functions $G_{1, k}\left(\sigma_{1} \zeta(w)\right)$ is uniquely determined by the coefficients of the asymptotic IR expansion, namely

$$
\lim _{w \rightarrow \infty} G_{1, k}\left(\sigma_{1} \zeta(w)\right)=u_{1, k}^{(0)}
$$

If one uses Eqs. (25) and (27) for $k=1,2$ in the previous expression one finds the following identities which relate the transport coefficients determined from linear response theory with the asymptotic regime of the function $G_{1, k}\left(\sigma_{1} \zeta(w)\right)$ in the IR regime [30,31]:

$$
\begin{aligned}
\frac{\eta}{s} & =-\frac{3}{40} \lim _{w \rightarrow \infty} G_{1,1}\left(\sigma_{1} \zeta(w)\right), \\
\frac{T\left(\eta \tau_{\pi}-\lambda_{1}\right)}{s} & =-\frac{9}{80} \lim _{w \rightarrow \infty} G_{1,2}\left(\sigma_{1} \zeta(w)\right) .
\end{aligned}
$$

Thus, the transasymptotic matching procedure automatically allows us to generalize the concept of a transport coefficient beyond the linear regime by promoting the functions $G_{1, k}$ to be an effective transport coefficient $[30,31]$. For first- and second-order transport coefficients we have, respectively,

$$
\begin{aligned}
\left(\frac{\eta}{s}\right)_{r} & =-\frac{3}{40} G_{1,1}\left(\sigma_{1} \zeta(w)\right), \\
{\left[\frac{T\left(\eta \tau_{\pi}-\lambda_{1}\right)}{s}\right]_{r} } & =-\frac{9}{80} G_{1,2}\left(\sigma_{1} \zeta(w)\right) .
\end{aligned}
$$

As a result, a new physical picture for nonlinear transport emerges: Summing over all the nonperturbative sectors at each order of the IR expansion leads to an effective renormalization of the transport coefficients. Each transport coefficient exhibits a transient non-Newtonian behavior while relaxing toward its asymptotic value in the IR. This transient rheological behavior is described by a dynamical $R G$ flow equation. This result extends earlier work in the context of the Boltzmann equation in the relaxation time approximation [30,31]. We emphasize that Eqs. (37) are modified when including higher-order moments as we shall explain in Sec. IV B 2.

Numerical results for the renormalized transport coefficients in Eqs. (37) are shown in Fig. 3. In order to obtain these results we have to calculate the functions $G_{1, k}$ with $k=\{1,2\}$ using Eq. (34). In that expression the transmonomials are given by Eqs. (31) and the coefficients $u_{1, k}^{(n)}$ are numerically determined by solving order by order the recursive relation (32). Figure 3 shows the $w$ dependence of the ratios between the dynamically renormalized transport coefficient over its asymptotic value. The top panel shows the shear viscosity over entropy density ratio, and the bottom panel shows the second-order transport coefficients.
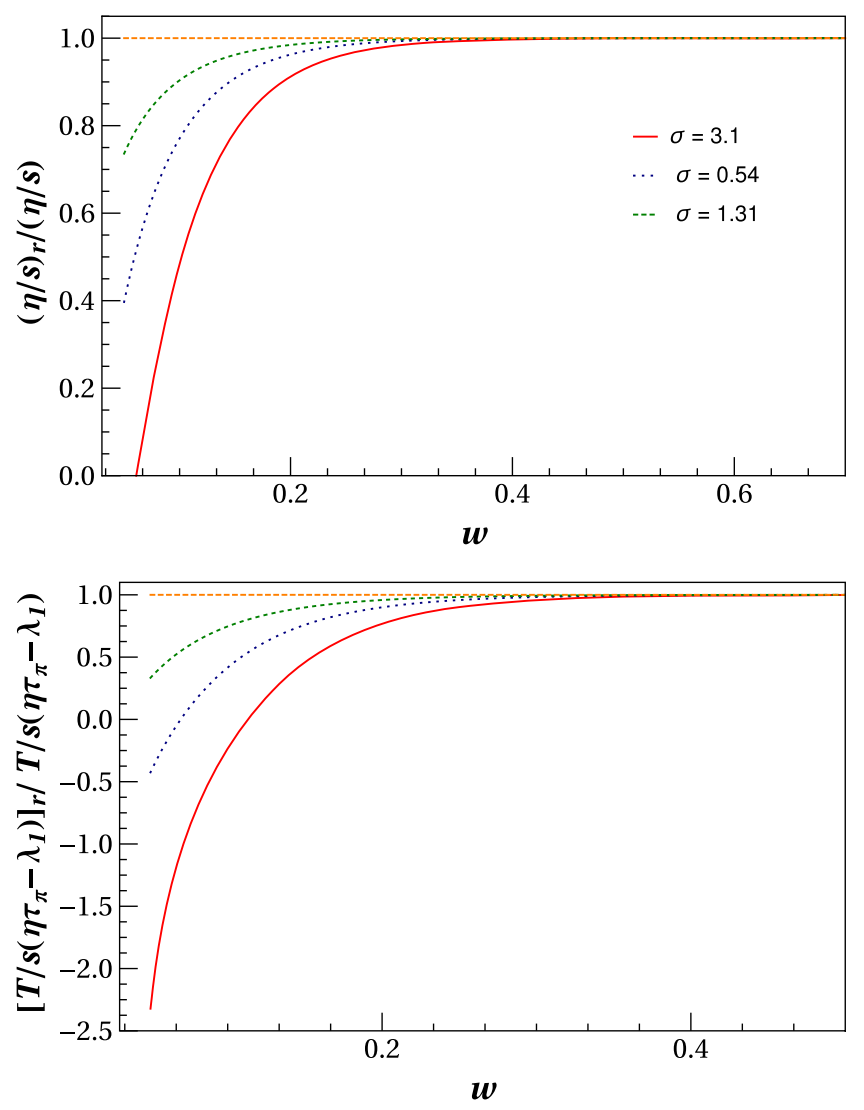

FIG. 3. Ratio of the dynamically renormalized transport coefficient over its corresponding asymptotic value as a function of $w$ for the $l=1$ case and for different initial conditions. Top and bottom panels correspond to the ratios of $(\eta / s)_{r} /(\eta / s)$ and $\left[(T / s)\left(\eta \tau_{\pi}-\lambda_{1}\right)\right]_{r} /\left[(T / s)\left(\eta \tau_{\pi}-\lambda_{1}\right)\right]$, respectively. See text for further details.

The values of the transasymptotic parameters were determined by fitting the transseries solution (31) truncated at $k=3, n=15$ to the numerical solution of the ODE for $c_{1}$ (23a) computed for $\theta_{0}=1$. The initial condition for $c_{1}$ was chosen as $c_{1}\left(w_{0}=0.05\right)=\{6.60119,0,-3.02976\}$. The corresponding best fit transseries parameters are $\sigma=\{3.1,1.31,0.54\}$. Since the determination of the transseries parameter was carried out by using a numerical least-square fit $[31,32]$ it leads to a highly nonlinear relation between $\sigma$ and $c_{1}\left(w_{0}\right)$ so both parameters are not proportional to each other.

Both panels illustrate that regardless of the initial condition each renormalized transport coefficient reaches its asymptotic value as it is expected from the properties of transasymptotic functions $G_{1, k}$ in Eq. (36). On the other hand, prior to the relaxation to the asymptotic values both renormalized transport coefficients feature a transient phase which depends on the deformation history of the fluid and, thus, its rheology. During this transient phase the transport coefficients increase monotonically which is expected mainly for two reasons: First, at the early stages the 

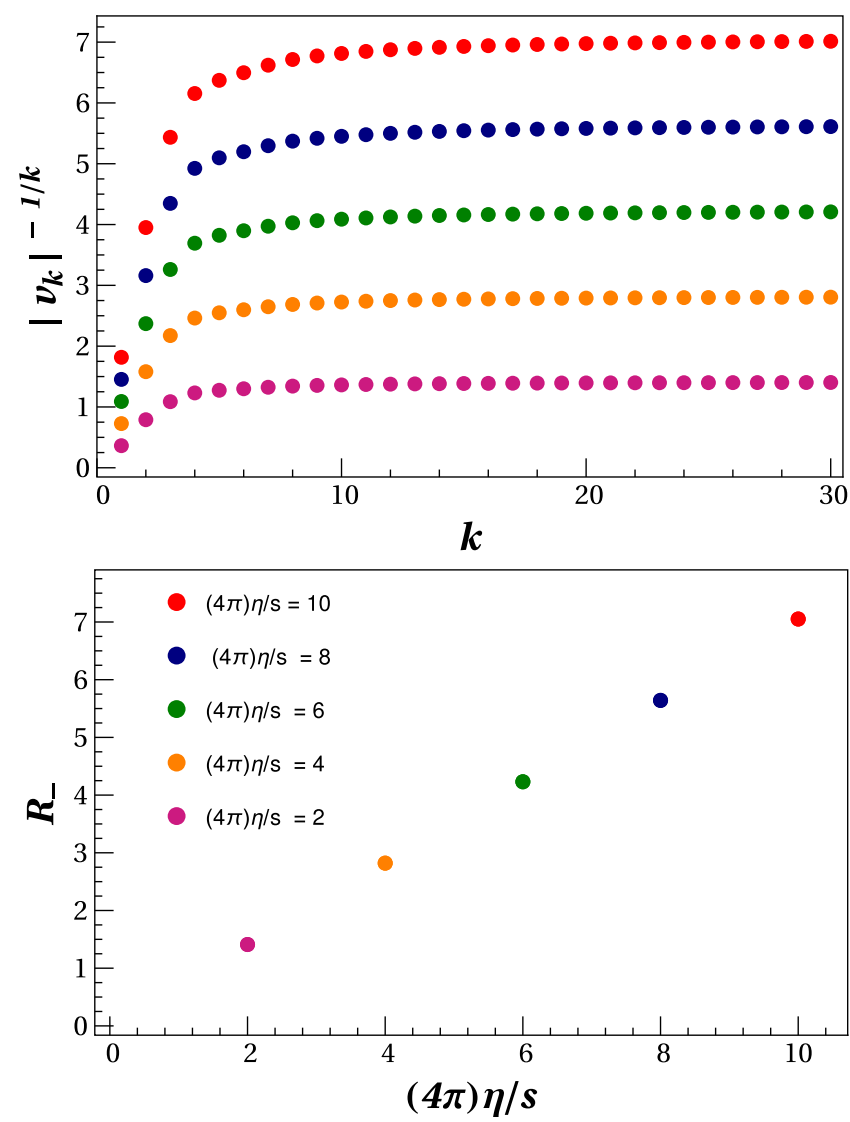

FIG. 4. Top: $\left|v_{k}\right|^{-1 / k}$ vs $k$ for different values of $(4 \pi) \eta / s=$ $\{2,4,6,8,10\}$ for magenta, orange, green, blue, and red dots, respectively. Bottom: Numerical value of the radius of convergence vs $\eta / s$. In this case the UV perturbative series expansion (45) is carried out around the UV fixed point $\bar{c}_{1}^{-}=(25-3 \sqrt{505}) / 14$.

expansion rate is larger than the collisional rate such that the effective transport coefficients get reduce compared with their corresponding asymptotic values. On the other hand, as the system hydrodynamizes, entropy production always increases so transport coefficients do the same until their values reached out their asymptotic values determined by linear response. Clearly the transient phase is not universal since it depends on the initial condition. However, one observes that both ratios shown in Fig. 4 saturate their value to the unity around $w \approx 0.4$. These results are consistent with the ones shown in Fig. 2. It is important to mention that the effective value of the renormalized transport coefficients change when increasing the number of moments [30,31]. We shall come back to this subtlety issue in Sec. IV B.

\section{Dynamical RG flow equation}

When the transasymptotic matching condition is imposed over the entire domain of $w$, a set of nonlinear PDEs for the functions $G_{1, k}$ is obtained after inserting Eq. (33) into the original ODE (23a),

$$
\begin{aligned}
& \left(-S_{1} \hat{\zeta}+\Lambda(1)\right) G_{1, k}+\left(-\beta_{1} \hat{\zeta}-k+\frac{12}{7}\right) G_{1, k-1}+4 \delta_{k, 1} \\
& \quad-\frac{1}{20} \sum_{k_{1}, k_{2}=0}^{k_{1}+k_{2}=k}\left[\left(-S_{1} \hat{\zeta}-\frac{40 \hat{\kappa}}{7}\right) G_{1, k_{1}}\right. \\
& \left.\quad+\left(-\beta_{1} \hat{\zeta}-k_{1}+5\right) G_{1, k_{1}-1}\right] G_{1, k_{2}} \\
& \quad+\frac{2 \hat{\kappa}_{1}}{15} \sum_{k_{1}, k_{2}, k_{3}=0}^{k_{1}+k_{2}+k_{3}=k} G_{1, k_{1}} G_{1, k_{2}} G_{1, k_{3}}=0,
\end{aligned}
$$

where $\hat{\zeta}=\zeta \partial / \partial \zeta=\partial / \partial \log \zeta$. Solving these PDEs is equivalent to summing over all fluctuation contributions around the IR. More importantly, these PDEs can be reinterpreted as $\mathrm{RG}$ flow equations for the transport coefficients given the identities (36). This statement can be rigorously proven within the gradient descent approach to the RG flows of quantum field theories in the context of dynamical systems, cf. [30,31,56]. Furthermore, a RG flow picture from a global point of view holds for our original dynamical system (22) provided the existence of an effective potential for the ODEs, also known as the Lyapunov function. Its mathematical existence is proven in Appendix D.

Following Refs. [30,31] the ODE for $c_{1}$ in terms of the $w$ variable, Eq. (23a) can be rewritten as

$$
\begin{gathered}
\frac{d c_{1}}{d \log w}=\beta_{1}\left(c_{1}, w\right), \\
\beta_{1}\left(c_{1}, w\right)=-\frac{1}{1-\frac{c_{1}}{20}}\left\{4+\frac{5}{7} c_{1}-\frac{1}{5} c_{1}^{2}\right. \\
\left.+\left[1+\frac{\pi^{2}}{\kappa}+\frac{2}{7} c_{1}+\frac{2}{15} c_{1}^{2}\right] \hat{\kappa} w c_{1}\right\},
\end{gathered}
$$

where $\hat{\kappa}$ is given in Eq. (20). The previous equation is the RG equation for the moment $c_{1}$ and the function $\beta_{1}\left(c_{1}, w\right)$ encodes the dependence on the RG time $w$. The variable $w \sim K n^{-1}$ plays the analogous role of the energy scale in Quantum Field Theory (QFT) since it determines the size of deviations from the equilibrium state.

We can think of the fugacity $\zeta_{1}$ as independent of $w$ [87], so that $G_{1, k}\left(\sigma_{1} \zeta_{1}\right)$ can be regarded as a simple coefficient in the transseries solution of $c_{1}$ (31). Then, the following identity follows [30,31]:

$$
\begin{aligned}
& \hat{\zeta}_{1} G_{1, k}\left(\sigma_{1} \zeta_{1}\right) \\
& =-\frac{1}{2 \pi i} \oint_{|w| \ll 1} d w \frac{w^{k-1}}{\left(S_{1} w+b_{1}\right)} \\
& \quad \times\left[\sum_{k^{\prime}=0}^{\infty} k^{\prime} G_{k^{\prime}}\left(\sigma_{1} \zeta_{1}\right) w^{-k^{\prime}}+\beta_{1}\left(c_{1}\left(\sigma_{1} \zeta_{1}, w\right), w\right)\right],
\end{aligned}
$$


where we explicitly used Eq. (39a). ${ }^{6}$ The Cauchy integral formula in the previous expression simply picks up the $k$ th coefficient of the term $\hat{\zeta}_{1} c_{1}$.

In order to determine the RG equation which describes the transient behavior of the transport coefficients we consider an arbitrary observable $\mathcal{O}=\mathcal{O}\left(G_{1, k}\left(\sigma_{1} \zeta_{1}(w)\right)\right)$. Its change along $w$ results in the following $\mathrm{RG}$ equation:

$$
\begin{aligned}
& \frac{d \mathcal{O}\left(G_{1, k}\left(\sigma_{1} \zeta_{1}(w)\right)\right)}{d \log w} \\
& =-\sum_{k=0}^{\infty}\left[\left(b_{1}+S_{1} w\right) \hat{\zeta}_{1} G_{1, k}\left(\sigma_{1} \zeta_{1}\right)\right] \frac{\partial \mathcal{O}}{\partial G_{1, k}} .
\end{aligned}
$$

The renormalization of the observable $\mathcal{O}$ is obtained by solving the previous equation. In this equation the term $\hat{\zeta}_{1} G_{1, k}\left(\sigma_{1} \zeta_{1}\right)$ is given by the identity (40) which encodes the dynamics of the original ODE via the beta function $\beta_{1}$ (39a). By setting $\mathcal{O}=-\frac{3}{40} G_{1}$ and $\mathcal{O}=-\frac{9}{80} G_{2}$ in Eq. (41) one determines the RG flow equations for the transport coefficients $\eta / s$ and $\frac{T\left(\eta \tau_{\pi}-\lambda_{1}\right)}{s}$, respectively. We point out to the reader that the $\mathrm{RG}$ flow equation is sensitive to the number of moments involved in any truncation scheme and its more generic form is derived in Sec. IV B.

\section{Transseries solutions in the UV}

In the previous section we showed that perturbations decay exponentially in the vicinity of the IR fixed point (29). For an arbitrarily chosen ODE linear perturbations do not necessarily decay exponentially in the neighborhood of a fixed point. For instance, consider a parameter $x \in(0, \infty)$ and a function $y(x)$ which satisfies a well-defined ODE $d y(x) / d x=\mathcal{L}(x, y(x))$. If this ODE has a source fixed point $y_{0}$ in the limit $x \rightarrow 0$, i.e., the Lyapunov exponent $\lambda$ close to $y_{0}$ is positive in this limit, then the solutions in a vicinity around it behave as $y(x) \sim e^{\lambda x} \sim \sum_{n=0}^{\infty}(\lambda x)^{n}$ in the limit $x \ll 1$. This approximate solution is a power-law series with a finite radius of convergence which can be extended by analytic continuation.

The stability analysis of the RTA Boltzmann equation indeed showed that, in the limit when the Knudsen number is large, that is in the $w \rightarrow 0$ limit, power-law series solutions for the Legendre moments emerge [31]. Similar findings were reported in [57-59]. The power series expansion is rather different from nonhydrodynamic modes in the gradient expansion (24). As we shall see in this section the main differences can be inferred from the

${ }^{6}$ The ordinary derivative respect to $\log w$ was rewritten as

$$
\frac{d}{d \log w}=-\left(b_{1}+S_{1} w\right) \hat{\zeta}_{1}+\frac{\partial}{\partial \log w} .
$$

In this loop integration, $\zeta_{1}$ is temporarily regarded as an independent variable on $w$. behavior of linearized perturbations around the UV and IR fixed points, respectively. The case studied here illustrates the importance of the flow structure in phase space in controlling the functional form of solutions for generic dynamical systems of ODEs [32]. In the following we will explain more carefully the emergence of the power series behavior in the case of the Fokker-Planck equation.

\section{Perturbative power series in the $U V$}

The limit $w \rightarrow 0$ of Eq. (23a) can be understood by changing variables $w \rightarrow 1 / z$ in Eq. (23). We obtain the following differential equation for $c_{1}(z)$ :

$$
\begin{gathered}
\frac{d c_{1}}{d z}=F_{1}\left(c_{1}, z\right), \\
F_{1}\left(c_{1}, z\right)=\frac{1}{\left(1-\frac{1}{20} c_{1}\right)}\left[\frac{1}{z}\left(4+\frac{5}{7} c_{1}-\frac{1}{5} c_{1}^{2}\right)\right. \\
\left.+\frac{1}{z^{2}}\left[\lambda(1) c_{1}+\frac{2 \hat{\kappa}}{7} c_{1}^{2}+\frac{2 \hat{\kappa}}{15} c_{1}^{3}\right]\right] .
\end{gathered}
$$

In the limit $z \rightarrow \infty$ the dominant term in the previous expressions is $\mathcal{O}\left(z^{-1}\right)$. It is straightforward to see that the fixed points correspond to the roots of the polynomial that multiplies the $\mathcal{O}(1 / z)$ term in Eq. (42b),

$$
4+\frac{5}{7} \bar{c}_{1}-\frac{1}{5} \bar{c}_{1}^{2} \equiv 0
$$

The UV fixed points are given by

$$
\bar{c}_{1}^{ \pm}=(25 \pm 3 \sqrt{505}) / 14=\left\{\begin{array}{l}
6.60119 \text { if }+, \\
-3.02976 \text { if }-.
\end{array}\right.
$$

In analogy to what we did in the IR limit one can construct a perturbative expansion in the limit $z \rightarrow \infty$. We consider

$$
c_{1}(z)=\sum_{k=1}^{\infty} \frac{v_{1, k}}{z^{k}}
$$

where $v_{1,0}=\bar{c}_{1}^{ \pm}$. A similar power series expansion was discussed in the case of kinetic models undergoing Gubser flow in $[29,32]$. Inserting the UV series expansion in Eq. (45) into Eq. (42a) we obtain the following recursive relation for the coefficients $v_{1, k}$ : 


$$
\begin{aligned}
& \left(\frac{2}{7}-k\right) v_{1, k-1}-4 \delta_{k, 1}-\lambda(1) v_{1, k-2} \\
& +\frac{1}{20} \sum_{k_{1}, k_{2}=0}^{k_{1}+k_{2}=k}\left[\left(k_{1}+3\right) v_{1, k_{1}-1}\right] v_{1, k_{2}} \\
& -\frac{2 \hat{\kappa}^{k_{1}+k_{2}=k-2}}{7} \sum_{k_{1}, k_{2}=0}^{v_{1, k_{1}}} v_{1, k_{2}}-\frac{2 \hat{\kappa}^{k_{1}+k_{2}+k_{3}=k-2} \sum_{k_{1}, k_{2}, k_{3}=0} v_{1, k_{1}} v_{1, k_{2}} v_{1, k_{3}}=0,}{}
\end{aligned}
$$

where $v_{1, k} \equiv 0$ if and only if $k<0$ and $v_{1,0}=\bar{c}_{1}^{ \pm}$.

\section{Stability analysis and radius of convergence}

When performing asymptotic expansions it is important to check the stability of the perturbative expansion. We address this issue for the UV power series expansion (45) by analyzing the linearized perturbations around it. Linearizing Eq. (42b) around the UV fixed points, i.e., $c_{1} \rightarrow \bar{c}_{1}^{ \pm}+\delta c_{1}^{ \pm}$, one finds the following evolution equation for the linearized perturbations in the $z \rightarrow \infty$ limit:

$$
\begin{aligned}
\frac{d \delta c_{1}^{ \pm}}{d z} & =\left.\frac{\partial F_{1}}{\partial c_{1}}\right|_{c_{1}=\bar{c}_{1}^{ \pm}} \delta c_{1}^{ \pm}+\mathcal{O}\left(\delta c_{1}^{ \pm} / z^{2}\right) \\
& \approx \frac{8 \sqrt{505}}{\sqrt{505} \mp 85} \frac{\delta c_{1}^{ \pm}}{z} .
\end{aligned}
$$

The solutions are

$$
\begin{aligned}
\delta c_{1}^{ \pm}(z) & =\mu_{1}^{ \pm} z^{\alpha_{1}^{ \pm}}, \\
\alpha_{1}^{ \pm} & =\frac{8 \sqrt{505}}{\sqrt{505} \mp 85} \equiv\left\{\begin{array}{l}
-2.87516 \text { if }+, \\
1.67278 \text { if }-
\end{array}\right.
\end{aligned}
$$

where $\mu_{1}^{ \pm}$is an integration constant associated with the UV fixed points. The power-law behavior of the linearized perturbations (48) is completely different from the behavior in the IR limit (29). We note that the exponents $\alpha_{1}^{ \pm}$do not depend on the strength of the coupling, but they are uniquely defined by the location of the UV fixed points. In the limit $z \rightarrow \infty$ the solution $\delta c_{1}^{+}=\mu^{+} z^{-2.87516}$ follows a power-law decay. The linear perturbation $\delta c_{1}^{-}=$ $\mu^{-} z^{1.67278}(48)$ monotonically increases. This divergence implies that the associated fixed point only admits a power series of the form (47) which can be extended analytically as it is shown below. Alternatively one may say that the initial condition $\mu_{1}^{-}$for the fluctuation around $\bar{c}_{1}^{-}$vanishes exactly, and thus the only possible expansion around $c_{1}^{-}$is a power series.

The formal power series in the $z$ variable (45) has a finite radius of convergence which can be estimated via the
Cauchy-Hadamard theorem ${ }^{7}$ [88]. We proceed to calculate the radius of convergence of the power series expansion (45) by considering first the expansion around $\bar{c}_{1}^{-}$. The case around $\bar{c}_{1}^{+}$is analyzed separately at the end of this section. The radius of convergence $R_{-}$is calculated by numerically solving the recursive relation (46). In Fig. 4 (top panel) we show the coefficients of the UV expansion $\left|v_{k}\right|^{-1 / k}$ vs the order of the expansion $k$ for different values of $(4 \pi) \eta / s=\{2,4,6,8,10\} .^{8}$ The coefficients $\left|v_{k}\right|^{-1 / k}$ stabilize for $k \geq 25-30$. This result confirms that the power series (47) has a finite radius of convergence. We analyze its dependence on the value of $\eta / s$ in Fig. 4 (bottom panel). This plots shows that $R_{-}$depends linearly on the value of the shear over entropy ratio $\eta / s$. The empirical relation between these two quantities extracted from this plot is

$$
R_{-}=0.70515\left(4 \pi \frac{\eta}{s}\right)+b_{-}
$$

where the intercept $b_{-}>0$ is very small $\mathcal{O}\left(10^{-8}\right)$ and consistent with zero. We verify the extracted numerical value of the radius of convergence by increasing $k$ up to $k_{\max }=100$. We found that the relative difference between the saturated bound $\left|v_{k}\right|^{-1 / k}$ for $k=25$ and $k=100$ was only $0.1 \%$.

The linear growth of the radius of convergence as a function of $\eta / s$ is intuitively understood as follows: If $\eta / s$ or, equivalently, the mean free path are large then the rate of collisions is small. As a result the Yang-Mills plasma will expand freely for a longer period of time. This finding might provide an explanation for the partial success of phenomenological models at intermediate scales of momentum larger than the typical temperature where the expansion is carried out in terms of a small number of scatterings [89-93].

In general the radius of convergence of a power series can be extended by analytical continuation [88]. The idea is to consider a power series of the form

\footnotetext{
${ }^{7}$ According to the Cauchy-Hadamard theorem [88], the radius of convergence $R$ of the formal power series of a function $f(z)$ around the point $a$ (with $b_{k}, a \in \mathfrak{E}$ )

$$
f(z)=\sum_{k=0}^{\infty} b_{k}(z-a)^{k}
$$

is given by

$$
R=\left[\lim _{n \rightarrow \infty} \sup \cdot\left|b_{n}\right|^{1 / n}\right]^{-1} .
$$

${ }^{8}$ The asymptotic value of $\eta / s$ and $\theta_{0}$ can be established via Eq. (27) which leads to the following identity:

$$
\frac{\eta}{s}=\frac{\theta_{0}}{5\left(\kappa+\pi^{2}\right)},
$$

where we used explicitly Eqs. (20).
} 


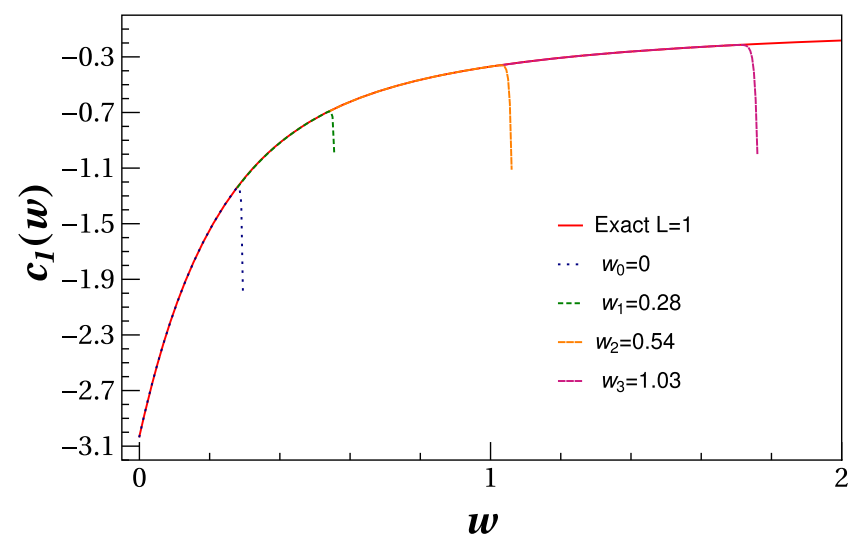

FIG. 5. Analytical continuation of the perturbative expansion for $c_{1}(w)$ in the UV around the fixed point $\bar{c}_{1}^{-}=(25-3 \sqrt{505}) / 14$. We show the exact solution as we perturbative expansions around the indicated values of $w$.

$$
c_{1}(w)=\sum_{k=0}^{\infty} v_{1, k}\left(w-w_{0}\right)^{k}
$$

where both $v_{1, k}$ and $w_{0} \in \Re, v_{1,0}=c_{1}\left(w_{0}\right)$ and in this case $w_{0} \geq 0$. The coefficients $v_{1, k}$ are determined explicitly by plugging Eq. (50) into the ODE (23a) and finding the associated recursive relation analogous to Eq. (46) which is now evaluated at $w=w_{0}$. By construction the series (50) has a finite radius of convergence $R_{0}$ so the series will diverge outside of $\left|w-w_{0}\right|<R_{0}$. One can thus expand again the function at another point $w_{1}$ located within a distance $R_{0}-\delta$ (with $\delta>0$ ) centered at $w_{0}$ and determine the new coefficients of the power series. Successively applying this approach one is extending the original power series significantly beyond its original radius of convergence $R_{0}$. We apply this method to our solution by initializing the expansion at $w_{0}=0$ around $\bar{c}_{1}^{-}$and $\theta_{0}=$ 1 (i.e., extremely low values of $\eta / s \rightarrow 0$ ). The agreement between the exact numerical result and the analytical continuation of the perturbative expansion in the UV is truly remarkable, as shown in Fig. 5.

\section{Resummation of the fluctuations around $\bar{c}_{1}^{+}$}

The power-law decay of linearized perturbations $\delta c_{1}^{+}$in Eq. (47), together with the UV stability analysis, strongly suggests a resummation scheme around the UV fixed point $\bar{c}_{1}^{+}$. The form of the equation in the $z$ variable, see Eq. (42a), resembles the corresponding results obtained in the case of the RTA Boltzmann equation for Bjorken and Gubser flows [31,32]. Following these approaches we consider the following UV transseries ansatz:

$$
c_{1}=\sum_{k=0}^{\infty} \sum_{n=0}^{\infty} v_{1, k}^{(n)} \varphi_{k}^{n},
$$

where the UV transmonomial is

$$
\varphi_{k}^{n}:=z^{-k}\left[\xi_{1}(z)\right]^{n}, \quad \xi_{1}(z)=\mu_{1} z^{\alpha_{1}}
$$

with $\alpha_{1}=-2.86516$. We note that, unlike the IR transseries where fluctuations decay exponentially close to the fixed point, the transmonomials $\xi_{1}(z)$ (52) are non-negligible close to the UV fixed point. For instance, the first fluctuation term $z^{\alpha_{1}} \approx z^{-2.87516}$ is comparable to terms of order $\mathcal{O}\left(z^{-3}\right)$. This implies that the complete solution near $\bar{c}_{1}^{+}$requires a double summation, over both the perturbative exponent $k$ as well as the transmonomials $(52)$ [31,32]. We also observe that the approximate UV solution (51) has a finite radius of convergence even after resumming the fluctuations around $\bar{c}_{1}^{+}$[32]. Finally, in the UV regime it is not possible to implement IR transasymptotic matching (see Sec. III B) since the solution (51) is not valid for all values of $z$ given the finite radius of convergence.

The coefficients $v_{1, k}^{(n)}$ in Eq. (51) are determined by inserting this ansatz into Eq. (42a). We obtain the recursion relation

$$
\begin{aligned}
& \left(n \alpha_{1}-k+\frac{2}{7}\right) v_{1, k-1}^{(n)}-4 \delta_{n, 0} \delta_{k, 1}-\lambda(1) v_{1, k-2}^{(n)} \\
& -\frac{1}{20} \sum_{n_{1}, n_{2}=0}^{n_{1}+n_{2}=m} \sum_{k_{1}, k_{2}=0}^{k_{1}+k_{2}=k}\left[\left(n_{1} \alpha_{1}-k_{1}-3\right) v_{1, k_{1}-1}^{\left(n_{1}\right)}\right] v_{1, k_{2}}^{\left(n_{2}\right)} \\
& -\frac{2 \hat{\kappa}^{\prime}}{7} \sum_{n_{1}, n_{2}=0}^{n_{1}+n_{2}=n} \sum_{k_{1}+k_{2}=k-2}^{k_{1}, k_{2}=0} v_{1, k_{1}}^{\left(n_{1}\right)} v_{1, k_{2}}^{\left(n_{2}\right)} \\
& -\frac{2 \hat{\kappa}_{1}}{15} \sum_{n_{1}+n_{2}, n_{3}=0}^{n_{2}+n_{3}=n} \sum_{k_{1}+k_{2}+k_{3}=k-2} v_{k_{1}, k_{2}, k_{3}=0}^{\left(n_{1}\right)} v_{1, k_{1}}^{\left(n_{2}\right)} v_{1, k_{2}}^{\left(n_{3}\right)}=0 .
\end{aligned}
$$

For the UV transseries (51) the coefficients $v_{1,0}^{(0)}=\bar{c}_{1}^{+}$and the normalization of the integration constant is $v_{1,0}^{(1)}=1$.

\section{TRANSASYMPTOTIC ANALYSIS: THE GENERAL CASE}

In this section we generalize the transasymptotic analysis outlined in the previous section to the case when we consider the full vector of Legendre moments $\bar{c}=$ $\left(c_{1}, \ldots, c_{L}\right)^{T}$ with $L \geq 1$. We briefly outline how to generate IR and UV transseries solutions for the nonautonomous dynamical system (23). The techniques presented in the section were extensively discussed in [30-32], and we will focus on issues specific to the Fokker-Planck equation. More rigorous mathematical aspects of the techniques outlined in this section can also be found in Refs. [52,54].

\section{A. Transseries solutions in the IR}

We seek to find multiparameter transseries solutions to the ODEs (22) by following the generic procedure developed by Costin [52,54]. In the large $w$ limit and for the nonlinear ODEs (22) the Legendre moments admit IR expansions of the form $[30,31]$ 


$$
\bar{c}_{l}=\sum_{l=0}^{\infty} \frac{u_{l, k}}{w^{k}}
$$

By mathematical induction one can show that, by inserting the expansion (54) into Eq. (22), and considering only the linear regime of the ODE, then the leading large $w$ behavior of the moments $c_{l}$ is $c_{l} \sim \mathcal{O}\left(w^{-l}\right)$ [30,31]. These results can be used to linearize Eq. (22) by expanding around the IR expansion (54), i.e., $\mathbf{c} \rightarrow \overline{\mathbf{c}}+\delta \mathbf{c}$ where $\overline{\mathbf{c}}=\left(\bar{c}_{1}, \cdots \bar{c}_{L}\right)$. Here, each $\bar{c}_{l}$ is given by Eq. (54). By keeping only the leading terms $\mathcal{O}(1 / w)$ we get the following linearized equation for the IR perturbation $\delta \mathbf{c}$ :

$$
\begin{aligned}
& \frac{d \delta \mathbf{c}}{d w}=\left.\sum_{l=1}^{L} \frac{\partial \mathbf{F}(\mathbf{c}, w)}{\partial c_{l}}\right|_{\mathbf{c}_{l}=\overline{\mathbf{c}}_{l}} \cdot \delta \mathbf{c}_{l}, \\
& \approx-\left[\Lambda+\frac{1}{w}\left(\overline{\mathfrak{X}}-\frac{8}{\Lambda(1)} \overline{\mathfrak{Y}}+\frac{\Lambda}{5 \lambda(1)}-\frac{\boldsymbol{\Gamma}}{20}\right)\right] \delta \mathbf{c} \\
&=-\left[\Lambda+\frac{1}{w} \mathfrak{W}\right] \delta \mathbf{c}, \\
& \mathfrak{W}=\overline{\mathfrak{X}}-\frac{8}{\lambda(1)} \overline{\mathfrak{Y}}+\frac{\Lambda}{5 \lambda(1)}-\frac{\Gamma}{20},
\end{aligned}
$$

where

$$
\overline{\mathfrak{Y}}=\hat{\kappa}\left(\begin{array}{ccccc}
\Omega_{111} & \Omega_{112} & & & \\
\Omega_{211} & \Omega_{212} & \Omega_{213} & & \\
& \ddots & \ddots & \ddots & \\
& & \Omega_{L-11 L-2} & \Omega_{L-11 L-1} & \Omega_{L-11 L} \\
& & & \Omega_{L 1 L-1} & \Omega_{L 1 L}
\end{array}\right) .
$$

We can check that the truncated $l=1$ linear equation (28) is a special case of Eq. (55). In order to solve the linearized equation (55) we perform a transformation in terms of the pseudomodes $\delta \tilde{\mathbf{c}}$ [52]

$$
\delta \tilde{\mathbf{c}}=\tilde{V}(w) \delta \mathbf{c}, \quad \tilde{V}(w):=\mathbf{1}_{L}+\frac{V}{w},
$$

where $V$ is a constant matrix. Thus, the equation of the pseudomodes $\delta \tilde{\mathbf{c}}$ is

$$
\begin{aligned}
\frac{d \delta \tilde{\mathbf{c}}}{d w} & =-\tilde{V}(w)\left[\Lambda+\frac{1}{w} \mathfrak{W}\right] \tilde{V}(w)^{-1} \delta \tilde{\mathbf{c}}(\hat{\tau})+O\left(\delta \tilde{\mathbf{c}} / w^{2}\right) \\
& =-\left[\Lambda+\frac{1}{w}(\mathfrak{W}+[V, \Lambda])\right] \delta \tilde{\mathbf{c}}(w),
\end{aligned}
$$

where the commutator $[A, B]:=A B-B A$ was introduced. One can take diag. $(V)=(0, \ldots, 0)$, and the other components can be chosen in such a way that one can diagonalize the matrix $\mathfrak{W}$ as follows:

$$
\mathfrak{W}+[V, \Lambda]: \mapsto \tilde{\mathfrak{W}}:=\operatorname{diag} .\left(\mathfrak{w}_{1}, \ldots, \mathfrak{w}_{L}\right) .
$$

Therefore, the solution of Eq. (58) is

$$
\delta \tilde{c}_{\ell}(w)=\sigma_{\ell} \frac{e^{-\lambda(\ell) w}}{w^{\mathfrak{w}_{\ell}}} \Rightarrow \delta c_{\ell}(w)=\sigma_{\ell} \frac{e^{-\lambda(\ell) w}}{w^{\mathfrak{w}_{\ell}}} .
$$

The Lyapunov exponents are the diagonal components of the matrix $\hat{\Lambda}$ (18a). These exponents govern the rate at which each mode relaxes toward its equilibrium value, $w_{l}^{*}=[\lambda(l)]^{-1}$. We observe that for larger value of $l$ relaxation is faster, and that there is a clear hierarchy of scales $w_{1}^{*}>w_{2}^{*}>\cdots>w_{L}^{*}$. For the FP equation the slowest nonhydro mode is $c_{1}$ which is proportional to the normalized shear viscous tensor component. A common misunderstanding in the literature is to assume that the existence of this hierarchy of scales determines immediately the full set of the slowest degrees of freedom of the physical system. Recent studies $[31,68]$ have shown that this assumption is incorrect since nonlinear mode-to-mode coupling among moments plays a relevant role close to the forward attractor, and thus the determination of the slow invariant manifold of the dynamical system is not uniquely determined by the mere existence of a hierarchy of scales [31,32] in Eq. (13). The eigenvalue $\mathfrak{w}_{\ell} \in \mathbb{R}^{+}$of the matrix $\hat{\mathfrak{S}}$ is the anomalous dimension of the linear perturbation $\delta c_{l}$. Their values $\mathfrak{w}_{\ell}$ depends explicitly of the coupling constant as well as the truncation order $L$ [30-32]; only in the limit $L \rightarrow \infty$ do their values coincide with the exact underlying microscopic theory. We note that $\mathfrak{w}_{\ell}$ is not a universal constant. Its value depends on the kinetic model and is sensitive to nonlinearities encoded in mode-to-mode couplings.

The general transseries solutions of the nonlinear coupled ODEs (22) are constructed by rewriting these equations in terms of the pseudomodes basis, i.e.,

$$
\tilde{\mathbf{c}}=\tilde{V}(w) \mathbf{c},
$$

where $\tilde{V}(w)$ is given in Eq. (57). Under this transformation the equation of the pseudomodes $\tilde{\mathbf{c}}$ is

$$
\frac{d \tilde{\mathbf{c}}}{d w}=\tilde{\mathbf{F}}(\tilde{\mathbf{c}}, w),
$$

$$
\begin{aligned}
\tilde{\mathbf{F}}(\tilde{\mathbf{c}}, w)= & -\frac{1}{\left(1-\frac{c_{1}}{20}\right)} \\
& \times\left[\frac{1}{w}(\tilde{\mathfrak{X}}(\mathbf{c}) \tilde{\mathbf{c}}+\tilde{\boldsymbol{\Gamma}}(w))+(\tilde{\Lambda}+\tilde{\mathfrak{Y}}(\mathbf{c})+\tilde{\mathfrak{Z}}(\mathbf{c})) \tilde{\mathbf{c}}\right] \\
& -\frac{1}{w^{2}} V \tilde{V}^{-1}(w) \tilde{\mathbf{c}},
\end{aligned}
$$

where $c_{1}(w)=\sum_{k=1}^{L} \tilde{V}_{1 k}^{-1}(w) \tilde{c}_{k}(w)$. We have denoted matrices in the pseudomode basis by $\tilde{M}(w) \equiv \tilde{V}(w) M \tilde{V}^{-1}(w)$ and the vector $\tilde{\boldsymbol{\Gamma}}(w) \equiv \tilde{V}(w) \boldsymbol{\Gamma}$. Having determined the 
linearized pseudomodes (60) we can write the solutions of the pseudomodes as multiparameter transseries

$$
\begin{gathered}
\tilde{c}_{\ell}(w)=\sum_{\mathbf{n} \in \mathbb{N}_{0}^{L}} \sum_{k=0}^{+\infty} \tilde{u}_{\ell, k}^{(\mathbf{n})} \Phi_{k}^{\mathbf{n}}, \\
\Phi_{k}^{\mathbf{n}}:=\left(\prod_{j=1}^{L} \sigma_{j}^{n_{j}} \zeta_{j}^{n_{j}}\right) w^{-k}, \quad \text { with } \quad \zeta_{j}:=\frac{e^{-S_{j} w}}{w^{\beta_{j}}},
\end{gathered}
$$

where $\mathbf{n}=\left(n_{1}, n_{2}, \ldots, n_{L}\right)$ is a vector where each component $n_{i}$ is a non-negative integer which labels the nonperturbative sectors of the pseudomodes, $\sigma_{i} \in \mathbb{C}$ is the integration constant, and $L \in \mathbb{N}$ is the truncation order. The term $\zeta_{j}$ in Eq. (63b) must match the linearized solutions (60), and thus it determines the IR data

$$
S_{j}=\lambda(j), \quad \beta_{j}=\mathfrak{w}_{j} .
$$

The set of ODEs for the pseudomodes (62a) satisfy the asymptotic and regular conditions needed in Costin's prescription [54], and thus it justifies mathematically why the exact solutions are indeed multiparameter transseries. Now, given the solutions for the pseudomodes (63) one obtains the ones corresponding to the Legendre moments $c_{l}$ as follows:

$$
\begin{gathered}
c_{\ell}(w)=\sum_{\ell^{\prime}=1}^{L} \tilde{V}_{\ell \ell^{\prime}}^{-1}(w) \tilde{c}_{\ell^{\prime}}(w)=\sum_{\mathbf{n} \in \mathbb{N}_{0}^{L}} \sum_{k=0}^{+\infty} u_{\ell, k}^{(\mathbf{n})} \Phi_{k}^{\mathbf{n}}, \\
\text { with } \quad u_{\ell, k}^{(\mathbf{n})}=\sum_{\ell^{\prime}=1}^{L} \sum_{k^{\prime}=0}^{k}(-1)^{k^{\prime}}\left[V^{k^{\prime}}\right]_{\ell \ell^{\prime}} \tilde{u}_{\ell^{\prime}, k-k^{\prime}}^{(\mathbf{n})} .
\end{gathered}
$$

The reality condition on the distribution function implies that the Legendre moments $c_{l}$ are real. Although the solutions for the pseudomodes $\tilde{c}_{l}$ (63a) are complex the reconstructed transseries solutions of $c_{l}(65 \mathrm{a})$ are real since the following conditions are satisfied $\forall j \geq 1$ [32]:

(1) $\lambda(j) \in \mathbb{R}^{+}$[with $\lambda_{j}$ given by Eq. (18a)] and $\lambda(j) \neq$ $\lambda\left(j^{\prime}\right)$ for any $j \neq j^{\prime}$.

(2) If $\beta_{j} \in \mathbb{R}^{+}$which implies $\sigma_{j} \in \mathbb{R}^{+}$.

(3) If $\beta_{j} \in \mathbb{C}$ then there should be a complex conjugate pair $\beta_{k}=\beta_{j}^{*}(j \neq k)$ which implies $\sigma_{k}=\sigma_{j}^{*}$.

Finally the coefficients $\tilde{u}_{\ell, k}^{(\mathbf{n})}$ are determined by inserting the transseries solution of the pseudomodes (63a) into its corresponding Eq. (62a). As a result one gets the following recursive relation:

$$
\begin{aligned}
& -(\mathbf{m} \cdot \mathbf{S}) \tilde{\mathbf{u}}_{k}^{(\mathbf{m})}-(\mathbf{m} \cdot \boldsymbol{\beta}+k) \tilde{\mathbf{u}}_{k-1}^{(\mathbf{m})}+\frac{1}{20} \sum_{\mathbf{m}_{1}, \mathbf{m}_{2}=\mathbf{0}}^{\mathbf{m}_{1}+\mathbf{m}_{2}=\mathbf{m}} \sum_{k_{1}, k_{2}=0}^{k_{1}+k_{2}=k}\left[\left(\mathbf{m}_{1} \cdot \mathbf{S}\right) \tilde{\mathbf{u}}_{k_{1}}^{\left(\mathbf{m}_{1}\right)}+\left(\mathbf{m}_{1} \cdot \boldsymbol{\beta}+k_{1}\right) \tilde{\mathbf{u}}_{k_{1}-1}^{\left(\mathbf{m}_{1}\right)}\right] u_{1, k_{2}}^{\left(\mathbf{m}_{2}\right)} \\
& +[\{\tilde{\Lambda}+\tilde{\mathfrak{Y}}(\mathbf{u})+\tilde{\mathfrak{Z}}(\mathbf{u})\} \star \tilde{\mathbf{u}}]_{k}^{(\mathbf{m})}+[\tilde{\mathfrak{X}}(\mathbf{u}) \star \tilde{\mathbf{u}}+\tilde{\boldsymbol{\Gamma}}]_{k-1}^{(\mathbf{m})}+V \mathbf{u}_{k-2}^{(\mathbf{m})}=0,
\end{aligned}
$$

where $\tilde{\mathbf{u}}_{k}^{(\mathbf{m})}$ and $\mathbf{u}_{k}^{(\mathbf{m})}$ are coefficients of $\tilde{\mathbf{c}}$ and $\mathbf{c}$, respectively, $\left[\boldsymbol{\bullet}_{k}^{(\mathbf{m})}\right.$ denotes the coefficient for the basis $\Phi_{k}^{\mathbf{m}}$, which can be projected out by the loop integrations as

$$
a_{k}^{(\mathbf{m})}=\oint_{|w| \ll 1} \frac{d w}{2 \pi i} w^{k-1}\left[\prod_{\ell=1}^{L} \oint_{\left|\zeta_{\ell}\right| \ll 1} \frac{d \zeta_{\ell}}{2 \pi i} \frac{\zeta_{\ell}^{-m_{\ell}-1}}{\sigma_{\ell}^{m_{\ell}}}\right] A,
$$

with $\quad A=\sum_{\mathbf{m} \in \mathbb{N}_{0}^{L}} \sum_{k=0}^{\infty} a_{k}^{(\mathbf{m})} \Phi_{k}^{\mathbf{m}}$,

and $\star$ denotes the convolution product summing over $\mathbf{m}$ and $k$, equipping the usual matrix-vector product as well, defined as

$$
\begin{aligned}
{[a \star b]_{k}^{(\mathbf{m})} } & =\sum_{\mathbf{m}^{\prime} \geq \mathbf{0}}^{\mathbf{m}} \sum_{k^{\prime}=0}^{k} a_{k^{\prime}}^{\left(\mathbf{m}^{\prime}\right)} b_{k-k^{\prime}}^{\left(\mathbf{m}-\mathbf{m}^{\prime}\right)}, \\
\text { with } A & =\sum_{\mathbf{m} \in \mathbb{N}_{0}^{L}} \sum_{k=0}^{\infty} a_{k}^{(\mathbf{m})} \Phi_{k}^{\mathbf{m}}, \quad B=\sum_{\mathbf{m} \in \mathbb{N}_{0}^{L}} \sum_{k=0}^{\infty} b_{k}^{(\mathbf{m})} \Phi_{k}^{\mathbf{m}} .
\end{aligned}
$$

Here, we replaced the $\mathbf{c}$ dependence in the matrices with $\mathbf{u}$, and the multiplication between c (and also $w$ ) should be replaced with the convolution product. Notice that $\tilde{\boldsymbol{\Gamma}}_{k}^{(\mathbf{m})}$ is nonzero when $\mathbf{m}=\mathbf{0}$ and $k=0,1$.

\section{B. Transasymptotic matching: General case}

In this section we present the transasymptotic matching condition for the multiparameter transseries solutions of the dynamical system (22). The rigorous mathematical demonstration of the generalized transasymptotic matching condition can be found in Refs. [52,54]. In this general case it is more convenient to use the pseudomode basis defined in Eq. (61). Following the same arguments outlined in Sec. III B the solutions of the pseudomodes $\tilde{c}_{i}$ (with $i=1, \ldots, L)$ can be written as

$$
\tilde{c}_{i}(w)=\sum_{k \geq 0}^{\infty} \tilde{G}_{i, k}(\boldsymbol{\sigma} \zeta(w)) w^{-k},
$$

$$
\text { with } \quad \tilde{G}_{i, k}=\sum_{\mathbf{n} \in \mathbb{N}_{0}^{L}} \tilde{u}_{i, k}^{(\boldsymbol{n})}\left(\prod_{j=1}^{L} \sigma_{j}^{n_{j}} \zeta_{j}^{n_{j}}\right) .
$$


The transasymptotic matching condition (69a) can be directly obtained from (66) by taking the summation of $\mathbf{m}$ after putting $\prod_{\ell=1}^{L} \sigma^{m_{\ell}} \zeta^{m_{\ell}}$ in of the each terms, and it leads to the following first-order PDE for the functions $\tilde{\mathbf{G}}_{k} \equiv\left(\tilde{G}_{0, k}, \tilde{G}_{1, k}, \ldots, \tilde{G}_{L, k}\right)$ :

$$
\begin{aligned}
& -(\mathbf{S} \cdot \hat{\boldsymbol{\zeta}}) \tilde{\mathbf{G}}_{k}-(\boldsymbol{\beta} \cdot \hat{\boldsymbol{\zeta}}+k) \tilde{\mathbf{G}}_{k-1}+\frac{1}{20}\left[G_{1} \star(\mathbf{S} \cdot \hat{\boldsymbol{\zeta}}) \tilde{\mathbf{G}}\right]_{k}+\frac{1}{20}\left[G_{1} \star(\boldsymbol{\beta} \cdot \hat{\boldsymbol{\zeta}}) \tilde{\mathbf{G}}\right]_{k-1}+\frac{1}{20} \sum_{k_{1}, k_{2}=0}^{k_{1}+k_{2}=k} k_{1} \tilde{\mathbf{G}}_{k_{1}-1} G_{1, k_{2}} \\
& \quad+[\{\tilde{\Lambda}+\tilde{\mathfrak{Y}}(\mathbf{G})+\tilde{\mathfrak{Z}}(\mathbf{G})\} \star \tilde{\mathbf{G}}]_{k}+[\tilde{\mathfrak{X}}(\mathbf{G}) \star \tilde{\mathbf{G}}+\tilde{\boldsymbol{\Gamma}}]_{k-1}+V \mathbf{G}_{k-2}=0,
\end{aligned}
$$

where $[\bullet]_{k}$ denotes the coefficient for the basis $w^{-k}$, and $\star$ denotes the convolution product summing only over $k$. Notice that $\tilde{\boldsymbol{\Gamma}}_{k}=\tilde{\boldsymbol{\Gamma}}_{k}^{(\mathbf{0})}$. The functions $\tilde{G}_{i, k}$ depend only on $\sigma_{i} \zeta_{i}$. The solution of this equation gives us automatically the time evolution of any operator $\mathcal{O}\left(\tilde{c}_{i}\right)$ by including all the transmonomials. If one increases the number of moments it is not a trivial task to solve Eq. (70). In principle, Eq. (70) is a nonlinear PDE which can be solved provided a well-defined initial condition as well as a correct choice for the eigenbasis (see Ref. [52] for technical details). To the best of our knowledge, the only case known in the literature where the transasymptotic matching condition has been solved explicitly is the $l=1$ case for the RTA Boltzmann equation [30,31]. However, one can always reconstruct the functions $G_{i, k}$ from their definition 69)b) provided previous knowledge of the coefficients $\tilde{u}_{i, k}^{(\boldsymbol{n})}$ which are determined via the recursion relation (66). The information of the IR data is encoded in the integration constants $\sigma_{i}$ which in principle can be determined from the UV data.

\section{Dynamical RG flow equation: General case}

In this section we generalize the procedure of the RG flow equation when the number of moments $c_{l}$ is $l \geq 1$. Consider an observable $\mathcal{O} \equiv \mathcal{O}(\boldsymbol{c}(w))$ with $\boldsymbol{c}=\left(c_{1}, \ldots, c_{L}\right)$. Starting from the original ODE for the moments $c_{l}$ (22) and using Eq. (62a), one finds that the change of $\mathcal{O}$ along the $\mathrm{RG}$ time $w$ is

$$
\begin{aligned}
\frac{d \mathcal{O}(\boldsymbol{c}(w))}{d \log w} & =-\sum_{i=1}^{L}\left[\sum_{k=0}^{\infty}(\tilde{\mathbf{b}}+w \boldsymbol{S}) \cdot \hat{\zeta} \tilde{G}_{i, k}+k \tilde{G}_{i, k}\right] \frac{\partial \mathcal{O}}{\partial \tilde{c}_{i}}, \\
& =\sum_{i=1}^{L} \tilde{\beta}_{i} \cdot \frac{\partial \mathcal{O}}{\partial \tilde{c}_{i}},
\end{aligned}
$$

where $\tilde{\mathbf{F}}=\tilde{V} \mathbf{F}$ being the matrix $\tilde{V}$ the inverse of $\tilde{V}^{-1}$ in Eq. (61) [see also Eq. (62a)] and $\tilde{\beta}=w \tilde{\mathbf{F}}$. In the previous equation we use that $\frac{d}{d \log w}=(\tilde{\mathbf{b}}+w \boldsymbol{S}) \cdot \hat{\boldsymbol{\zeta}}+\frac{\partial}{\partial \log w}$ and we denote $\hat{\zeta}_{i}=\partial / \partial \log \zeta_{i}$. The first line of Eq. (71) encodes the scaling behavior of the observable $\mathcal{O}$ while the second one encodes the dynamics of the nonlinear ODEs through $\beta$. By solving this RG equation one sums over all the nonperturbative contributions of the multiparameter transseries and thus determines the renormalization of $\mathcal{O}$.

We are seeking a RG flow equation of the transport coefficients so it is more convenient to consider that $\mathcal{O}=\mathcal{O}\left(G_{i, k}\right)$. As a result, the transport coefficients depend intrinsically only on $\zeta(w)$. Thus, for this case the RG flow equation of $\mathcal{O}$ reads as follows:

$\frac{d \mathcal{O}\left(G_{i, k}(w)\right)}{d \log w}=-\sum_{i=1}^{L}\left[\sum_{k=0}^{\infty}(\tilde{\mathbf{b}}+w \boldsymbol{S}) \cdot \hat{\boldsymbol{\zeta}} \tilde{G}_{j, k}\right] \cdot \frac{\partial \mathcal{O}}{\partial \tilde{G}_{j, k}}$.

At this level the connection with the beta function $\tilde{\boldsymbol{\beta}}$ is highly nontrivial and thus, one must consider particular cases like the $l=1$ case studied in Sec. III B (see also Refs. [30,32] for further examples).

\section{Renormalization of first- and second-order transport coefficients}

From the results derived in the previous section we have the tools to study qualitatively the effect of adding more moments on the renormalized transport coefficients. In general, it is proven that the asymptotic leading-order behavior of the moments $c_{l} \sim \mathcal{O}\left(w^{-l}\right)$. The perturbative Chapman-Enskog expansion up to second order [78] leads to the following asymptotic series expansion of the moments $c_{1}$ and $c_{2}$ as follows $[31,63,94]$ :

$$
\begin{gathered}
c_{1}=-\frac{40}{3} \frac{1}{w} \frac{\eta}{s}-\frac{80}{9} \frac{1}{w^{2}} \frac{T\left(\eta \tau_{\pi}-\lambda_{1}\right)}{s}+\mathcal{O}\left(1 / w^{3}\right), \\
c_{2}=\frac{80}{9} \frac{T\left(\lambda_{1}+\eta \tau_{\pi}\right)}{s} \frac{1}{w^{2}}+\mathcal{O}\left(1 / w^{3}\right) .
\end{gathered}
$$

If we now implement the transasymptotic matching condition (69a) together with the asymptotic expressions of the moments we conclude

$$
\frac{\eta}{s}=-\frac{3}{40} \lim _{w \rightarrow \infty} G_{1,1}(\sigma \zeta(w)),
$$

$$
\begin{gathered}
\frac{T}{s} \eta \tau_{\pi}=-\frac{9}{160} \lim _{w \rightarrow \infty}\left(G_{1,2}(\sigma \zeta(w))-G_{2,2}(\sigma \zeta(w))\right), \\
\frac{T}{s} \lambda_{1}=\frac{9}{160} \lim _{w \rightarrow \infty}\left(G_{1,2}(\sigma \zeta(w))+G_{2,2}(\sigma \zeta(w))\right) .
\end{gathered}
$$


The previous expressions generalize the results derived for the $l=1$ case, Eqs. (37). It is then straightforward to implement the newly developed concept of nonequilibrium transport coefficients when adding more moments as follows:

$$
\begin{gathered}
\left(\frac{\eta}{s}\right)_{r}=-\frac{3}{40} G_{1,1}(\boldsymbol{\sigma \zeta}(w)) \\
\left(\frac{T}{s} \eta \tau_{\pi}\right)_{r}=-\frac{9}{160}\left(G_{1,2}(\boldsymbol{\sigma \zeta}(w))-G_{2,2}(\boldsymbol{\sigma \zeta}(w))\right), \\
\left(\frac{T}{s} \lambda_{1}\right)_{r}=\frac{9}{160}\left(G_{1,2}(\boldsymbol{\sigma \zeta}(w))+G_{2,2}(\boldsymbol{\sigma} \zeta(w))\right) .
\end{gathered}
$$

The RG evolution equations of the first- and second-order transport coefficients are automatically determined by using the previous expressions and replacing $\mathcal{O} \rightarrow \mathcal{O}_{i}^{r}$ into Eq. (72) where $\mathcal{O}_{i}^{r}=\left((\eta / s)_{r},\left((T / s) \eta \tau_{\pi}\right)_{r},\left((T / s) \lambda_{1}\right)_{r}\right)$. These equations also illustrate that the inclusion of more moments indeed affects the values of the renormalized transport coefficients. One can also determine the renormalized transport coefficient by solving the recursive relation (66) and then reconstructing the multiparameter transseries as it was done in Fig. 3. However, the anomalous dimension $\beta_{i}$, the coefficients $u_{l, k}^{(\boldsymbol{n})}$, and the integration constants $\sigma_{i}$ [see Eq. (31)] become very sensitive to the number of moments added [31,32]. Furthermore, the number of integration constants $\sigma$ 's increase so the uncertainty to compare the IR and UV data becomes more cumbersome [31,32]. The only terms that are insensitive to the changes in the number of dynamical variables are the Lyapunov exponents $\lambda(l)$. In general, any truncation scheme would lead to a multiparameter transseries with a finite radius of convergence (see Appendix D in Ref. [31] and Sec. 3.3. in Ref. [32]). A possible way to circumvent this problem was proposed recently [95] where the ratio between the inverse Reynolds number and the Knudsen number was determined numerically.

\section{Transseries solutions in the UV}

In this section we derive the UV transseries solutions by generalizing the results of Sec. III C 1. We show that the linear perturbations of the Legendre modes $c_{l}$ around the UV fixed point, which is a source, follow a powerlaw decay. We begin by discussing some features of the stability of the dynamical system of ODEs (22c) in the UV regime.

Consider the $w \rightarrow 0$ limit of Eq. (22c) by first changing the variable $w=1 / z$. We get

$$
\frac{d \mathbf{c}}{d z}=\mathbf{F}(\mathbf{c}, z),
$$

$\mathbf{F}(\mathbf{c}, z)=\frac{1}{\left(1-\frac{c_{1}}{20}\right)}\left[\frac{1}{z}(\mathfrak{X}(\mathbf{c}) \mathbf{c}+\boldsymbol{\Gamma})+\frac{1}{z^{2}}(\hat{\Lambda}+\mathfrak{Y}(\mathbf{c})+\mathfrak{Z}(\mathbf{c})) \mathbf{c}\right]$.

In the $z \rightarrow \infty$ limit the dominant terms of the previous equation are $\mathcal{O}(1 / z)$. In this limit one can determine the fixed points by solving the following equation:

$$
\mathfrak{X}(\mathbf{c}) \mathbf{c}+\boldsymbol{\Gamma}=0 .
$$

The solutions to this equation provides a set of vectors $\overline{\mathbf{c}}=\left(\bar{c}_{1}, \ldots, \bar{c}_{L}\right)$ which determine the UV fixed points of the dynamical system. The solutions to Eq. (77) depend on the truncation order $l_{\max }$, so these are not necessarily real and we need to impose the reality conditions. Furthermore, the original FPE admits two UV fixed points for the moment $c_{1}$, which have the property that the transverse and longitudinal pressures are minimized, $c_{1}=5$ and $c_{1}=-5 / 2$, respectively. We call these configurations maximally prolate and maximally oblate, respectively. The two UV fixed points act as bounds for the basin of attraction from above and below along the $c_{1}$ ray in the infinite dimensional space of the moments $c_{l}$. Thus, any truncation scheme of the distribution function can be considered a good approximate solution of the Boltzmann equation if and only if the UV stability properties are reproduced approximately. In this case, the truncated dynamical system of ODEs should have at least two UV fixed points for the moment $c_{1}$.

In Fig. 6 we plot the real solutions to Eq. (77) for the moment $\bar{c}_{1}$ at the fixed points as a function of the truncation order $l_{\max } \in[1,10]$. We find that if $l_{\max }$ is odd then the real solutions for $\bar{c}_{1}$ come in pairs. As $l_{\max }$ increases (for odd values) the UV fixed points approach the expectation for the original FPE, $\bar{c}_{1}=\{5,-5 / 2\}$. On the other hand, there is only one real solution for $\bar{c}_{1}$ if $l_{\max }$ is even.

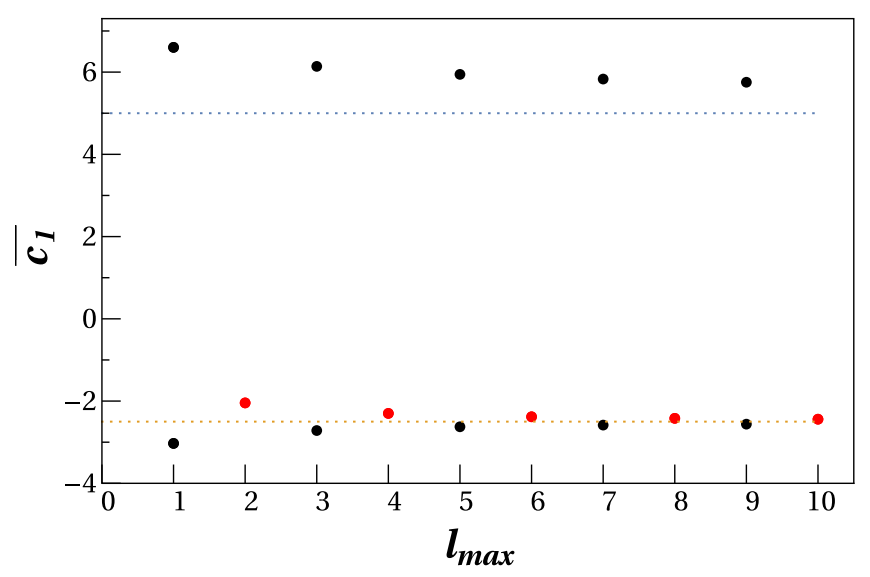

FIG. 6. Distribution of the UV fixed points for the moment $\bar{c}_{1}$ as a function of the truncation order $l_{\max }$. Black and red dots correspond to $l_{\max }$ being either an odd or an even number, respectively. Blue $\left(\bar{c}_{1}=5\right)$ and brown $\left(\bar{c}_{1}=-5 / 2\right)$ dashed lines correspond to the UV fixed points of the original FPE. 
These observations also hold for the UV fixed points of higher-order moments $\bar{c}_{l}$. Thus, the standard idea of obtaining a more accurate approximate solution to the Boltzmann equation by adding more moments to the distribution function is not necessarily correct. A good truncation scheme must reproduce the flow structure of the original Boltzmann equation in the IR and UV regimes. Similar findings were reported for the RTA Boltzmann equation [31,32]. In the rest of this section we will consider the case that $l_{\max }$ is odd.

In the limit $z \rightarrow \infty$ we linearized Eq. (76) around a particular UV real fixed point $\overline{\mathbf{c}}$, namely $\mathbf{c} \rightarrow \overline{\mathbf{c}}+\delta \mathbf{c}$, which leads to the following equation for the linear perturbations:

$$
\begin{aligned}
\frac{d \delta \mathbf{c}}{d z} & =\left.\sum_{k=1}^{L} \frac{\partial \mathbf{F}(\mathbf{c}, w)}{\partial c_{k}}\right|_{c_{k}=\bar{c}_{k}} \delta c_{k} \\
& =\frac{1}{1-\frac{\bar{c}_{1}}{20}}\left[\overline{\mathfrak{X}}-\frac{\bar{c}_{1}}{5} \mathbf{1}_{L}-\frac{1}{5}\left(\begin{array}{cccc}
\bar{c}_{1} & 0 & \cdots & 0 \\
\bar{c}_{2} & 0 & \cdots & 0 \\
\vdots & \vdots & \ddots & \vdots \\
\bar{c}_{L} & 0 & \cdots & 0
\end{array}\right)\right]\left(\frac{\delta \mathbf{c}}{z}\right) \\
& \equiv \mathfrak{I}(\overline{\mathbf{c}}) \cdot\left(\frac{\delta \mathbf{c}}{z}\right),
\end{aligned}
$$

where the matrix $\mathfrak{X}$ is defined in Eq. (16a). Upon inspection we find that Eq. (47) is a particular case of this linearized equation for the perturbations. We introduce the linear pseudomodes $\delta \tilde{\mathbf{c}}=\mathfrak{U} \delta \mathbf{c}$ so that $\mathfrak{U}$ diagonalizes the matrix $\mathfrak{I}(\overline{\mathbf{c}})$,

$$
\begin{aligned}
& \mathfrak{T}(\overline{\mathbf{c}}) \rightarrow \mathfrak{U} \mathfrak{T}(\overline{\mathbf{c}}) \mathfrak{U}^{-1} \equiv \tilde{\mathfrak{I}}(\overline{\mathbf{c}}) \\
& \text { with } \quad \tilde{\mathfrak{I}}(\overline{\mathbf{c}})=\operatorname{diag} .\left(\hat{\mathfrak{t}}_{1}, \ldots, \hat{\mathfrak{t}}_{L}\right) .
\end{aligned}
$$

This procedure gives the equation for the linearized pseudomodes

$$
\frac{d \delta \tilde{\mathbf{c}}}{d z}=\frac{1}{z} \tilde{\mathfrak{T}}(\overline{\mathbf{c}}) \cdot \delta \tilde{\mathbf{c}}
$$

Its solution is

$$
\begin{gathered}
\delta \tilde{c}_{i}=\mu_{i} z^{\hat{\mathrm{t}}_{i}}, \\
\Rightarrow \delta c_{i}=\mathfrak{U}_{i j}^{-1} \delta \tilde{c}_{j}=\mathfrak{U}_{i j}^{-1} \mu_{j} w^{-\hat{\mathfrak{t}}_{j}} .
\end{gathered}
$$

This result shows that close to the UV fixed points the linearized perturbations have a power-law behavior which is opposite to their IR counterparts (60). In general the eigenvalue $\hat{\mathfrak{t}}_{i}$ and the integration constant $\mu_{i}$, as well as the matrix element $\mathfrak{U}_{i j}$ are complex numbers. The reality condition of the linearized modes is satisfied since the coefficients of the polynomial eigenvalue problem of the matrix $\mathfrak{I}$ are real, and thus, their complex eigenvectors and eigenvalues come in complex conjugate pairs. When summing over the complete eigenbases of pseudomodes the linearized modes are real as expected. Moreover, what happens in the vicinity of any UV fixed point $\overline{\mathbf{c}}$ along the ray $\bar{c}_{i}$ is encoded by the real part of the eigenvalues of the matrix $\mathfrak{I}$, namely $\mathfrak{R}\left(\hat{\mathfrak{t}}_{i}\right)$. In the limit $z \rightarrow \infty$ the linearized perturbation $\delta \tilde{c}_{i}$ decays rapidly as a power law if $\Re\left(\hat{\mathfrak{t}}_{i}\right)<0$ while $\delta \tilde{c}_{i}$ increases when $\mathfrak{R}\left(\hat{\mathfrak{t}}_{i}\right)>0$.

In order to generate approximate UV solutions in the limit $z \rightarrow \infty$ limit it is convenient to rewrite the equations of motion in terms of the pseudomode basis $\tilde{\mathbf{c}}_{i}=\mathfrak{U}_{i j} \mathbf{c}_{j}$ [with $\mathfrak{U}$ given by Eq. (79)]. In terms of the pseudomodes the nonlinear ODEs (76) read

$$
\begin{gathered}
\frac{d \tilde{\mathbf{c}}}{d z}=\tilde{\mathbf{F}}(\tilde{\mathbf{c}}, z), \\
\tilde{\mathbf{F}}(\tilde{\mathbf{c}}, z)=\frac{1}{\left(1-\frac{c_{1}}{20}\right)}\left[\frac{1}{z}(\tilde{\mathfrak{X}}(\mathbf{c}) \tilde{\mathbf{c}}+\tilde{\boldsymbol{\Gamma}})+\frac{1}{z^{2}}(\tilde{\Lambda}+\tilde{\mathfrak{Y}}(\mathbf{c})+\tilde{\mathfrak{Z}}(\mathbf{c})) \tilde{\mathbf{c}}\right]
\end{gathered}
$$

In the previous equation it must be understood that $c_{1}=\sum_{j=1}^{L} \mathfrak{U}_{1 j}^{-1} \tilde{c}_{j}$. The transseries ansatz solution is built up based on the solutions of the linearized perturbations (81). These solutions can be taken as the transmonomials of the transseries which carry out the information about the nonperturbative contributions. Therefore, in the limit $z \rightarrow \infty$ the transseries ansatz is given by $[31,32]$

$$
\begin{gathered}
\tilde{c}_{l}(z)=\sum_{|\mathbf{m}| \geq 0}^{\infty} \sum_{k=0}^{\infty} \tilde{v}_{l, k}^{(\mathbf{m})} \boldsymbol{\varphi}_{k}^{\mathbf{m}}, \\
\boldsymbol{\varphi}_{k}^{\mathbf{m}}:=\frac{1}{z^{k}}\left(\prod_{j=1}^{L} \xi_{j}^{m_{j}}\right), \quad \text { with } \quad \xi_{j}=\mu_{j} z^{\hat{t}_{j}},
\end{gathered}
$$

where $\mathbf{m} \in \mathbb{N}_{0}^{L}, \mu_{i} \in \mathbb{C}$ is the integration constant and $L \in$ $\mathbb{N}$ is the truncation order. The UV data are determined by matching the transmonomial $\xi_{j}$ with the linearized solutions (81), and thus the anomalous dimensions $\alpha_{j}$ entering in the previous expression are the set of eigenvalues $\hat{\mathbf{t}}$ of the linearization matrix $\hat{\mathfrak{I}}$, Eq. (79), evaluated at the UV fixed points $\overline{\mathbf{c}}$. A similar transseries ansatz was proposed for the RTA Boltzmann equation in systems undergoing Bjorken [31] and Gubser flow [32]. The solutions of the Legendre moments in terms of the pseudomodes are given by

$$
\begin{gathered}
c_{l}(z)=\sum_{l^{\prime}=1}^{L} \mathfrak{U}_{l l^{\prime}}^{-1} \tilde{c}_{l^{\prime}}=\sum_{|m|=0}^{\infty} \sum_{k=0}^{\infty} v_{l, k}^{(\mathbf{m})} \boldsymbol{\varphi}_{k}^{\mathbf{m}}, \\
v_{l, k}^{(\mathbf{m})}=\sum_{r=1}^{L} \mathfrak{U}_{l r}^{-1} \tilde{v}_{r, k}^{(\mathbf{m})} .
\end{gathered}
$$


The coefficients $\tilde{v}_{l, k}^{(\mathbf{m})}$ entering in the transseries ansatz are determined entirely by inserting Eq. (83) into Eq. (82) which leads to the following recursive relation:

$$
\begin{gathered}
(\mathbf{m} \cdot \hat{\mathbf{t}}-k+1) \tilde{\mathbf{v}}_{k-1}^{(m)}-\frac{1}{20} \sum_{\mathbf{m}_{1}, \mathbf{m}_{2}=\mathbf{0}}^{\mathbf{m}_{1}+\mathbf{m}_{2}=\mathbf{m}} \sum_{k_{1}, k_{2}=0}^{k_{1}+k_{2}=k}\left[(\mathbf{m} \cdot \hat{\mathbf{t}}-k+1) \tilde{\mathbf{v}}_{k_{1}-1}^{\left(\mathbf{m}_{1}\right)}\right] v_{1, k_{2}}^{\left(\mathbf{m}_{2}\right)} \\
+[\{\tilde{\Lambda}+\tilde{\mathfrak{Y}}(\mathbf{u})+\tilde{\mathbf{Z}}(\mathbf{u})\} \star \tilde{\mathbf{u}}]_{k-2}^{(\mathbf{m})}+[\tilde{\mathfrak{X}}(\mathbf{u}) \star \tilde{\mathbf{u}}+\tilde{\boldsymbol{\Gamma}}]_{k-1}^{(\mathbf{m})}=0,
\end{gathered}
$$

where $\star$ denotes the convolution product. The normalization condition is chosen to be $\tilde{v}_{i, 0}^{(\mathbf{n})}=1$ when $n_{j}=\delta_{j, i}$ and the coefficients $\tilde{v}_{i, 0}^{(\mathbf{0})}=\mathfrak{U}_{i j} \bar{c}_{j}$ since one expands around a given UV fixed point $\overline{\mathbf{c}}$. In Eq. (86) $\tilde{\mathbf{v}}_{k}^{(\mathbf{m})}$ and $\mathbf{v}_{k}^{(\mathbf{m})}$ are coefficients of $\tilde{\mathbf{c}}$ and $\mathbf{c}$, respectively, and $[\bullet]_{k}^{(\mathbf{m})}$ denotes a coefficient proportional to $\varphi_{k}^{\mathbf{m}}$.

We emphasize that the transseries ansatz (83) is a convergent series with a finite radius of convergence. The rate of convergence depends on the anomalous dimension $\alpha_{i} \equiv \mathfrak{R}\left(\hat{t}_{i}\right)$ governing linearized perturbations $\delta \tilde{c}_{i}$ (81). The lessons learned from the $l=1$ truncation studied in Sec. III C (see also Refs. [31,32]) show that the power-law transseries solutions (84) can be constructed if and only if $\Re\left(\hat{t}_{i}\right)<0$. When $\Re\left(\hat{t}_{i}\right)>0$ the best option is to perform an analytical continuation while canceling the transmonomial contributions in Eq. (83) by setting the integration constant $\mu_{i} \equiv 0$.

\section{UNIVERSAL ASPECTS OF ATTRACTORS FOR DIFFERENT KINETIC MODELS}

In the previous sections we analyzed the behavior of the solutions of the FPE in both the UV and IR limits. In this section we study universal aspects of the attractors in the FPE as well as the RTA Boltzmann equation and the AMY kinetic theory [60].

Following the approach of Refs. [95,96] we consider a distribution function which is squeezed along the beam direction at early time. ${ }^{9}$ In this situation the initial longitudinal pressure vanishes exactly, and thus this configuration determines the pullback attractor of the distribution function at early times $[30,32]$. Under this condition the initial phase space distribution can be modeled as [95]

$$
f_{0}\left(\tau_{0}, p_{T}, p_{\varsigma}\right)=(2 \pi)^{3} \delta\left(p_{\varsigma}\right) \frac{d N_{0}}{d \varsigma d^{2} \mathbf{p}_{\mathbf{T}} d^{2} \mathbf{x}_{\mathbf{T}}}
$$

\footnotetext{
${ }^{9}$ For the RTA Boltzmann equation the form of the initial distribution function does not play a major role. However different processes of Yang-Mills plasmas, i.e., elastic and inelastic interactions, affect the parametrization of the momentum distributions [97,98]. In our approach these effects do not play a role at the level of the moments since the free streaming expansion dominates at early times over the collision rate of the FPE as discussed in Sec. IV C.
}

The normalization constant is chosen such that the initial energy density per unit rapidity per transverse area is constant

$$
\frac{d E_{0}}{d \varsigma d^{2} \mathbf{x}_{\mathbf{T}}}=\lim _{\tau_{0} \rightarrow 0} \tau_{0} e\left(\tau_{0}\right)=(\tau e)_{0}=\text { const. }
$$

The initial distribution function determines the initial conditions for the Legendre moments

$$
c_{\ell}=(4 l+1)(-1)^{l} \frac{\Gamma(l+1 / 2)}{\Gamma(l+1)} .
$$

Previous studies $[86,96]$ have shown that universal behavior of the numerical solutions can be by analyzed in terms of observables that are less sensitive to the initial conditions. Following Refs. [95,96] we study the following observable:

$$
\begin{aligned}
\mathcal{E} & =\frac{\tau^{4 / 3} \epsilon(\tau)}{\left(\epsilon \tau^{4 / 3}\right)_{\text {hydro }}}, \\
\left(\epsilon \tau^{4 / 3}\right)_{\text {hydro }} & \equiv \lim _{\tau \rightarrow \infty} \tau^{4 / 3} \epsilon(\tau) .
\end{aligned}
$$

We will analyze this observable as a function of the variable $\tilde{w}=\tau T(\tau) /[(4 \pi) \eta / s]$. In terms of this variable the results are insensitive to the strength of the coupling. Equation (90) has two interesting limits in the forward and pullback attracting regions. The former is described by a few terms of the nonhydrodynamic expansion [86], while the latter is determined by expanding around the UV fixed point where the longitudinal pressure vanishes [96],

$$
\mathcal{E}(\tilde{w})=\left\{\begin{array}{l}
C_{\infty}^{-1} \tilde{w}^{4 / 9} \text { (pullback attractor), } \\
1-\frac{2}{3 \pi} \frac{1}{\tilde{w}} \text { (forward attractor), }
\end{array}\right.
$$

where the constant $C_{\infty}$ is determined from Eq. (51) in Ref. [95]. Alternatively one can determine this constant by fitting to the numerical data. We verified that both methods lead to the same approximate value for this constant, see also $[95,96]$.

We numerically solved the evolution equations for the Legendre moments, Eqs. (14) and (21), respectively, together with the conservation law (14a). The solutions for the Legendre moments $c_{l}$ were obtained by truncating 


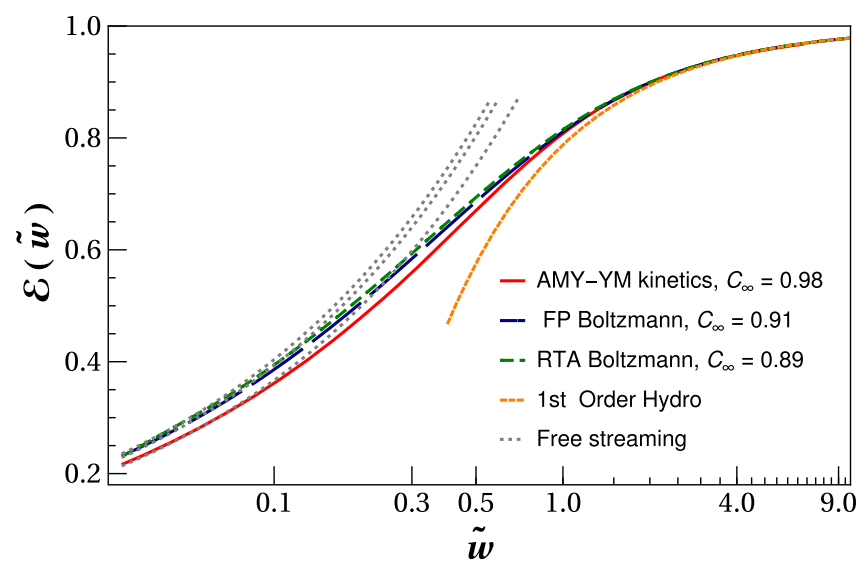

FIG. 7. Evolution of the normalized energy density (90) vs $\tilde{w}=\tau T(\tau) /[(4 \pi) \eta / s]$. We show the numerical results obtained for the FPE (dashed blue line), AMY-YM kinetics (red line), and RTA Boltzmann (dashed green line). In addition we present the universal hydrodynamic behavior (dashed orange line) and earlytime free streaming (dotted gray line).

the expansion at $l<l_{\max }$, setting $c_{l} \equiv 0$ for $l>l_{\max }$. This method converges rapidly and no sizable deviations are observed for $l_{\max } \gtrsim 35$ for both the RTA Boltzmann and FPE equations. In the case of the AMY-YM(Yang Mills) theory we used publicly available numerical results ${ }^{10}$ $[96,99,100]$.

In Fig. 7 we show numerical solutions of $\mathcal{E}$ as a function of $\tilde{w}$ for the RTA Boltzmann equation (dashed green line), FPE (dashed blue line), and AMY kinetic theory (red line). The general behavior of the different kinetic models is quite similar. They all exhibit a smooth transition from early-time free streaming to universal late-time hydrodynamic behavior. Deep in the UV regime the expansion (dashed gray lines) dominates over collisions regardless of the underlying collision kernel, and the pullback attractor is determined by free streaming expansion. A more surprising fact is that the interactions modify the expansion for similar values of $\tilde{w} \approx 0.08$, i.e., at a large Knudsen number $K n \approx 12.5$, independent of the underlying microscopic theory. We note that the existence of a pullback attractor is manifest only in the $w$ variable. In the original proper time variable $\tau$ the limit $\tau_{0} \rightarrow 0$ is not well defined because the ODEs have an essential singularity at this point [31,32].

In the UV regime the main difference among the kinetic models depicted in Fig. 7 is the values of the constant $C_{\infty}=$ $\{0.98,0.91,0.89\}$ for the AMY-YM, FPE, and RTA models, respectively. These values allows us to quantify the difference among the predictions of RTA and FPE with respect to the AMY-YM model. The former differs by $\sim 10 \%$ while the latter $\sim 8 \%$. It is somewhat surprising that a relatively simple approximation such as RTA captures

\footnotetext{
${ }^{10}$ The numerical data obtained in $[96,99,100]$ are available at [101]. We thank S. Schlichting for directing us to this site.
}

many aspects of the nonequilibrium dynamics compared with AMY-YM kinetics. This observation was also noticed previously in $[95,96]$.

In the IR regime we observe in Fig. 7 that all kinetic models reach the nonhydrodynamic behavior and thus, the forward attractor is encoded by the late-time nonhydrodynamic expansion. All the kinetic models reach the asymptotic hydrodynamic gradient expansion around $\tilde{w} \approx 2$, and thus $K n \approx 0.5$ so the deviations from equilibrium are quite large. This finding implies that one can reach the nonhydrodynamic behavior without having local thermal equilibrium.

Given that we find forward attractors for a number of different kinetic equations, one may ask how general this feature is. In Appendix E we discuss a sufficient set of mathematical conditions which ensure its existence for weakly coupled boost-invariant systems with highly nonlinear collision kernels beyond those studied in this work.

\section{DISCUSSION AND FINAL REMARKS}

In this work we studied the hydrodynamization of a boost-invariant system of gluons described by kinetic equation derived from QCD in the small-angle, diffusive approximation. We demonstrate that the physics of the Fokker-Planck equation can be recast in terms of a set of nonlinear ODEs for the Legendre moments of the oneparticle distribution function. We show that these kinetic equations admit transseries solutions in both the UV and IR regimes. These findings extend previous results obtained in the context of the Boltzmann equation in the relaxation time approximation [30-32].

We employ techniques from the theory of nonlinear dynamical systems. Applying these methods to the kinetic equations we investigate the stability properties of linearized perturbations around the IR and UV fixed points. We analyze the emergence of transseries solutions and show that their functional form is a rather natural consequence of the stability properties of the nonlinear ODEs around the UV and IR fixed points, respectively.

In the IR regime we prove that the solutions of the moments equations are multiparameter transseries. The associated transmonomials are built up using the behavior of linearized perturbations around the IR fixed point. These perturbations involve the product of an integration constant, an exponentially decaying term, and a power-law term. The exponentially decaying term determines the rate at which linearized perturbations decay near the IR fixed point. This decay time is controlled by the product of the Lyapunov exponent and the inverse Knudsen number. These terms play an analogous role of instantons contributions in QFT. The IR transseries effectively resums nonperturbative dissipative contributions that are absent in the usual perturbative gradient expansion. These results strongly suggest that a consistent formulation of nonhydrodynamic 
theories in far-from-equilibrium regimes requires the inclusion on nonperturbative physics.

In the IR regime the constitutive relations of each Legendre moment $c_{l}$ contain the nonperturbative information encoded in the multiparameter transseries. The result suggests a nonperturbative dynamic renormalization scheme which goes beyond standard linear response theory. In this theoretical framework, transport coefficients can be defined in far-from-equilibrium regimes when the Knudsen number is large. At each order of the perturbative IR expansion, transport coefficients are dynamically renormalized by nonperturbative corrections in the transseries, a method known as transasymptotic matching in the resurgence literature. This approach has a nice interpretation in terms of a RG flow.

From the physical point of view, the renormalized transport coefficients depend on the rheology of the fluid. They describe the relaxation of dissipative coefficients to their values dictated by the linear response approach. Thus, the fluid experiences transient non-Newtonian behavior prior to hydrodynamization.

On the mathematical side, we explored the relation between dynamical systems and RG flows. Indeed, it is known that the field theoretical RG flows may be discussed in the framework of autonomous dynamical systems $[55,56]$. Our work shows that this link can be extended to nonautonomous systems that hydrodynamize, where time plays the role of scale parameter. In other words, a nonautonomous dynamical system can be considered as a RG flow equation provided there is a slow invariant manifold, i.e., when the long-time physics is dominated by slow degrees of freedom [32]. In the case studied here and in [30-32] the invariant manifold is shown to exist if the IR perturbations go to zero in the long-time limit, a fact that points to the existence of a forward attractor. In particular, there are bounded solutions at $w \rightarrow \infty$ or $\tau \rightarrow$ $\infty$ for a fixed initial time that approach the equilibrium point. For expanding systems such as Gubser flow [29,32], the system is completely perturbative so that full hydrodynamization does not occur. Therefore, there is no slow invariant manifold, which in turn explains why the RG flow paradigm for the transport coefficients is no longer available.

In the UV regime the nonlinear ODEs for the moments admit transseries solutions with a rather different behavior compared to their counterparts in the IR limit. These solutions are power series with a finite radius of convergence. The radius of convergence $R$ grows linearly with the shear viscosity over entropy ratio $\eta / s$. The linear relation between $R$ and $\eta / s$ provides a rather simple explanation of previous numerical findings [72,75,76,102-108] where it was noticed that initial conditions close to the maximally oblate UV fixed point converge slowly to the forward nonhydrodynamic attractor than the ones located in the vicinity of the maximally prolate UV fixed point. The latter is a saddle point for any viable truncation (e.g., if the truncation bound for $l$ is odd) and it is found that there is a set of solutions for the Boltzmann equation that remain bounded near this point. In contrast, the maximally oblate UV fixed point is a source from which flow lines are streaming away in $w_{0} \rightarrow 0$ for a fixed $w$, meaning that there are no attracting regions to probe in the past of the dynamical system around this fixed point.

We show that the validity of the power series expansion can be extended by analytic continuation. As an alternative, we also introduce a new resummation scheme which accounts for the nonperturbative physics of the powerlaw behavior of the fluctuations around the UV fixed points. We note that the UV stability analysis also unveils a nontrivial aspect of truncating the moment expansion in relativistic kinetic theory: In order to preserve the UV structure of the original kinetic equation for Bjorken flow we have to include an odd number of Legendre moments $L=2 n+1$ moments in the $n \rightarrow \infty$ limit.

We discussed the general properties of the IR and UV regimes of the Fokker-Planck equation in comparison to the RTA Boltzmann and AMY kinetic equations. The pullback attracting region is entirely determined by the free streaming limit where the longitudinal extent of the distribution function is negligible. The attracting region in the long-time limit corresponds to hydrodynamization and can be characterized by a few terms in the gradient expansion. There are, however, minor differences between different models. We find that the RTA and AMY differ by at most $10 \%$ while the latter disagrees by up to $8 \%$ with the FPE result.

The techniques presented in this work are not necessarily restricted to far-from-equilibrium systems undergoing longitudinal boost-invariant expansion. Indeed, these methods can be applied to describe nonequilibrium dynamics in other physical systems of relevance such as cold atoms systems, condensed matter, and cosmology.

\section{ACKNOWLEDGMENTS}

We would like to thank M. Spalinśki, J. Jankowski, S. Schlichting, and A. Mazeliauskas for useful discussions. M. M. and T.S. are supported in part by the U.S. Department of Energy Award No. DE-FG0203ER41260. M. M., T. S., and V. S. are partially supported by the BEST (Beam Energy Scan Theory) DOE Topical Collaboration. S. K. is supported by the Polish National Science Centre Grant No. 2018/29/B/ST2/02457. A. B. was partially supported by DOE Award No. DESC0013036 and the National Science Foundation under Grant No. NSF PHY-1125915. V. S. acknowledges support by the DOE Office of Nuclear Physics through Award No. DE-SC0020081. V.S. thanks the ExtreMe Matter Institute EMMI (GSI Helmholtzzentrum für Schwerionenforschung, Darmstadt, Germany) for partial support and hospitality. 


\section{APPENDIX A: EVOLUTION EQUATIONS FOR THE LEGENDRE MOMENTS $c_{l}$}

In this Appendix we briefly describe the main elements to derive the evolution equation for the Legendre moments $c_{l}$. Some important identities of the Legendre polynomials were used and are explicitly listed at the end of this section, see A 1. The equation for the temperature is obtained from the conservation law which in our case reads as

$$
\frac{d \epsilon}{d \tau}+\frac{1}{\tau}\left(\epsilon+p_{L}\right)=0 \Rightarrow \frac{d T(\tau)}{d \tau}=-\frac{T(\tau)}{3 \tau}\left(1+\frac{1}{10} c_{1}(\tau)\right)
$$

where we used explicitly the matching condition for the energy (12a) with $c_{0} \equiv 1$. In order to obtain the equations for the Legendre moments $c_{l}$ we multiply first both sides of the FPE (1) by $\int_{p}(-u \cdot p)^{2} P_{2 l}\left(\cos \theta_{\mathbf{p}}\right)$. As a result we get

$$
\int_{\mathbf{p}}(-u \cdot p)^{2} P_{2 l}\left(\cos \theta_{p}\right) \partial_{\tau} f_{\mathbf{p}}=\lambda^{2} l_{C b} \int_{\mathbf{p}}(-u \cdot p)^{2} P_{2 l}\left(\cos \theta_{p}\right)\left\{\nabla_{\mathbf{p}} \cdot\left[\mathcal{J}(\tau) \nabla_{\mathbf{p}} f_{\mathbf{p}}+\mathcal{K}(\tau) \frac{\mathbf{p}}{p} f_{\mathbf{p}}\left(1+f_{\mathbf{p}}\right)\right]\right\} .
$$

Now we equate in the previous expression the ansatz (8) into the previous expression. In the lhs of Eq. (A2) one simply gets

$$
\begin{aligned}
\int_{\mathbf{p}}(-u \cdot p)^{2} P_{2 \ell}\left(\cos \theta_{p}\right) \frac{\partial f(\tau, \mathbf{p})}{\partial \tau}= & \int_{\mathbf{p}}(-u \cdot p)^{2} P_{2 \ell}\left(\cos \theta_{p}\right)\left[\sum _ { \ell ^ { \prime } = 0 } ^ { + \infty } \left(\frac{d c_{\ell^{\prime}}(\tau)}{d \tau} P_{2 \ell^{\prime}}\left(\cos \theta_{p}\right) f_{\mathrm{eq}}(\tau, \mathbf{p})\right.\right. \\
& \left.\left.+c_{\ell^{\prime}}(\tau) \frac{\partial P_{2 \ell^{\prime}}\left(\cos \theta_{p}\right)}{\partial \tau} f_{\mathrm{eq}}(\tau, \mathbf{p})+c_{\ell^{\prime}}(\tau) P_{2 \ell^{\prime}}\left(\cos \theta_{p}\right) \frac{\partial f_{\mathrm{eq}}(\tau, \mathbf{p})}{\partial \tau}\right)\right], \\
= & \frac{\epsilon(\tau)}{(4 \ell+1)}\left[\frac{d c_{\ell}(\tau)}{d \tau}+\frac{1}{\tau}\left\{\mathfrak{U}_{l} c_{\ell+1}(\tau)+\left(\mathfrak{B}_{l}-\frac{2}{15} c_{1}\right) c_{\ell}(\tau)+\mathfrak{c}_{l} c_{\ell-1}(\tau)\right\}\right],
\end{aligned}
$$

where we used explicitly the conservation law for the temperature (A1). In the previous expression the coefficients $\mathfrak{U}_{l}, \mathfrak{B}_{l}$, and $\mathfrak{\mathfrak { c }}_{l}$ are, respectively,

$$
\mathfrak{A}_{\ell}=-\frac{(2 \ell-1)(2 \ell+1)(2 \ell+2)}{(4 \ell+3)(4 \ell+5)}, \quad \mathfrak{B}_{l}=\frac{2\left(14 \ell^{2}+7 \ell-2\right)}{(4 \ell-1)(4 \ell+3)}-\frac{4}{3}, \quad \mathfrak{夭}_{\ell}=\frac{2 \ell(2 \ell-1)(2 \ell+2)}{(4 \ell-3)(4 \ell-1)} .
$$

The calculation of the momentum integrals in the rhs of Eq. (A2) simplifies if one replaces the ansatz (8) in the definition of the integrals $\mathcal{J}$ and $\mathcal{K}$, Eqs. (3), i.e.,

$$
\begin{gathered}
\mathcal{J}(\tau)=\int \frac{d^{3} p}{(2 \pi)^{3}} f(\tau, \mathbf{p})[1+f(\tau, \mathbf{p})]=\frac{T(\tau)^{3}}{2 \pi^{2}}\left[2 \zeta(3)+\sum_{n=0}^{+\infty} \frac{\pi^{2}-6 \zeta(3)}{3(4 n+1)} c_{n}(\tau)^{2}\right], \\
\mathcal{K}(\tau)=2 \int \frac{d^{3} p}{(2 \pi)^{3}} \frac{f(\tau, \mathbf{p})}{p}=\frac{T(\tau)^{2}}{6}
\end{gathered}
$$

where $\zeta(n)$ is the Riemann zeta function and the Landau matching condition for energy density (12a) was explicitly used.

In order to perform the momentum integrals in the rhs of Eq. (A2) we change the variable $p_{z}=p_{\varsigma} / \tau$. This results in changing the momentum measure $\frac{d^{2} \mathbf{p}_{T} d p_{\varsigma}}{\tau} \rightarrow d^{2} \mathbf{p}_{T} d p_{z}$ as well as the comoving energy $p^{\tau} \rightarrow p=\sqrt{\mathbf{p}_{\mathbf{T}}^{2}+p_{z}^{2}}$. Furthermore, this change of variable allows us to write the spatial components of the momentum as $p^{i}=p\left(\cos \phi_{p} \sin \theta_{p}, \sin \phi_{p} \sin \theta_{p}, \cos \theta_{p}\right)$. For instance, one of the integrals in the rhs of Eq. (A2) gives us

$$
\begin{aligned}
\int_{\mathbf{p}}(-u \cdot p)^{2} P_{2 l}\left(\cos \theta_{p}\right) \nabla_{\mathbf{p}}^{2} f(\tau, \mathbf{p})= & \int \frac{d^{3} p}{(2 \pi)^{3}} p P_{2 l}\left(\cos \theta_{p}\right) \\
& \times\left[\frac{\partial^{2}}{\partial p^{2}}+\frac{2}{p} \frac{\partial}{\partial p}+\frac{1}{p^{2}} \frac{\partial}{\partial \cos \theta_{p}} \sin ^{2} \theta_{p} \frac{\partial}{\partial \cos \theta_{p}}\right] f\left(\tau, p \sin \theta_{\mathbf{p}}, p \cos \theta_{\mathbf{p}}\right) \\
= & -\frac{T(\tau)^{2}}{6(4 \ell+1)}[\ell(2 \ell+1)-1] c_{\ell}(\tau) .
\end{aligned}
$$


In the previous expression we make explicit use of the Bjorken constraints over the total distribution function, i.e., $f_{\mathbf{p}}=f\left(\tau,\left|\mathbf{p}_{T}\right|, p_{\varsigma} / \tau\right) \rightarrow f\left(\tau, p \sin \theta_{\mathbf{p}}, p \cos \theta_{\mathbf{p}}\right)$ after the aforementioned change of variable is carried out.

The remaining momentum integral in the rhs of Eq. (A2) reads as

$$
\begin{aligned}
& \int_{\mathbf{p}}(-u \cdot p)^{2} P_{2 l}\left(\cos \theta_{p}\right) \nabla_{\mathbf{p}} \cdot \frac{\mathbf{p}}{p} f(\tau, \mathbf{p})(1+f(\tau, \mathbf{p})) \\
& \quad=-\frac{T(\tau)^{3}}{2 \pi^{2}(4 \ell+1)}\left[2 \zeta(3) c_{\ell}(\tau)+\left(\frac{\pi^{2}}{3}-2 \zeta(3)\right) \sum_{m, n=0}^{|m-n|=\ell} \Omega_{\ell m n} c_{m}(\tau) c_{n}(\tau)\right],
\end{aligned}
$$

where

$$
\Omega_{\ell m n}=\frac{\alpha_{m-n+\ell} \alpha_{n+m-\ell} \alpha_{n-m+\ell}}{\alpha_{n+m+\ell}} \cdot \frac{4 \ell+1}{2(n+m+\ell)+1}, \quad \text { with } \alpha_{\ell}=\frac{(2 \ell-1) ! !}{\ell !} .
$$

By equating Eqs. (A6) and (A7) together with Eqs. (A5) into the rhs in Eq. (A2) we finally get

$$
\begin{aligned}
\int_{\mathbf{p}}(-u \cdot p)^{2} P_{2 l}\left(\cos \theta_{p}\right) \mathcal{C}[f]= & -\frac{T(\tau) \epsilon(\tau)}{(4 \ell+1) \theta_{0}}\left(2 \zeta(3)+\sum_{n=0}^{+\infty} \frac{\pi^{2}-6 \zeta(3)}{3(4 n+1)} c_{n}(\tau)^{2}\right)(\ell(2 \ell+1)-1) c_{\ell}(\tau) \\
& -\frac{T(\tau) \epsilon(\tau)}{(4 \ell+1) \theta_{0}}\left[2 \zeta(3) c_{\ell}(\tau)+\left(\frac{\pi^{2}}{3}-2 \zeta(3)\right) \sum_{m, n=0}^{|m-n|=\ell} \Omega_{\ell m n} c_{m}(\tau) c_{n}(\tau)\right]
\end{aligned}
$$

with $\theta_{0}^{-1}=\frac{5}{8 \pi^{5}} \lambda_{Y M}^{2} l_{C b}$. Thus, by putting together the lhs (A3) and rhs (A9) of Eq. (A2) we get the following evolution equations for the Legendre moments $c_{l}$ :

$$
\begin{aligned}
\frac{d c_{\ell}(\tau)}{d \tau}= & -\frac{1}{\tau}\left[\mathfrak{A}_{\ell} c_{\ell+1}(\tau)+\left(\mathfrak{B}_{\ell}-\frac{2}{15} c_{1}(\tau)\right) c_{\ell}(\tau)+\mathfrak{\mho}_{\ell} c_{\ell-1}(\tau)\right] \\
& -\frac{T(\tau)}{\theta_{0}}\left[\left\{\kappa+\frac{\pi^{2} \ell(2 \ell+1)}{3}\right\} c_{\ell}(\tau)+\kappa \sum_{m, n=1}^{|m-n| \leq \ell} \Omega_{\ell m n} c_{m}(\tau) c_{n}(\tau)+\kappa \sum_{n=1}^{+\infty} \frac{(2 \ell-1)(\ell+1)}{3(4 n+1)} c_{n}(\tau)^{2} c_{\ell}(\tau)\right] .
\end{aligned}
$$

After some redefinition of the variables and writing them in a matrix form one gets Eqs. (14).

\section{Some useful identities of the Legendre polynomials}

In the previous section we used explicitly the following identities of the Legendre polynomials:

$$
\begin{gathered}
P_{n}(x)=2^{n} \sum_{k=0}^{n}\left(\begin{array}{l}
n \\
k
\end{array}\right)\left(\begin{array}{c}
\frac{n+k-1}{2} \\
n
\end{array}\right) x^{k}=\frac{1}{2^{n}} \sum_{k=0}^{n}\left(\begin{array}{l}
n \\
k
\end{array}\right)^{2}(x-1)^{n-k}(x+1)^{k}, \\
P_{n}(x) P_{m}(x)=\sum_{k=0}^{\operatorname{Min}[n, m]} \frac{\alpha_{m-k} \alpha_{k} \alpha_{n-k}}{\alpha_{n+m-k}} \cdot \frac{2(n+m-2 k)+1}{2(n+m-k)+1} \cdot P_{n+m-2 k}(x) . \quad \text { with } \alpha_{k}=\frac{(2 k-1) ! !}{k !}, \\
\int_{-1}^{+1} d x P_{2 n}(x) P_{2 m}(x)=\frac{2}{4 n+1} \delta_{n, m}, \\
\int_{-1}^{+1} d x P_{2 n}(x) P_{2 m}(x) P_{2 \ell}(x)=\frac{\alpha_{m-n+\ell} \alpha_{n+m-\ell} \alpha_{n-m+\ell}}{\alpha_{n+m+\ell}} \cdot \frac{2}{2(n+m+\ell)+1}, \\
x^{2} P_{2 \ell}(x)=\frac{2 \ell(2 \ell-1)}{(4 \ell-1)(4 \ell+1)} P_{2 \ell-2}(x)+\frac{8 \ell^{2}+4 \ell-1}{(4 \ell-1)(4 \ell+3)} P_{2 \ell}(x)+\frac{(2 \ell+1)(2 \ell+2)}{(4 \ell+1)(4 \ell+3)} P_{2 \ell+2}(x),
\end{gathered}
$$




$$
\begin{gathered}
\frac{\partial}{\partial \cos \theta_{p}} \sin ^{2} \theta_{p} \frac{\partial}{\partial \cos \theta_{p}} P_{2 \ell}\left(\cos \theta_{p}\right) \\
=-2 \ell(2 \ell+1) P_{2 \ell}\left(\cos \theta_{p}\right) .
\end{gathered}
$$

\section{APPENDIX B: ON THE INHERENT EXPONENTIAL ERROR}

We conclude this section by estimating the error made when truncating the transseries solutions based on superand hyperasymptotics [51]. In Sec. III A we observe a good agreement between the numerical results and the IR transseries solutions (see also Refs. [30-32]). Naively, one might think that a better agreement between the transseries and the numerical results can be obtained when adding more transmonomials and/or higher orders in the transseries. Nevertheless, this is not the case since in our case the radius of convergence of the transseries is finite $[31,32]$.

Consider the truncated IR asymptotic expansion of the Legendre mode $c_{1}$, i.e.,

$c_{1}(w) \sim \sum_{k=1}^{K} u_{1, k}^{(0)} w^{-k}+\mathcal{O}\left(w^{-K-1}\right), \quad$ with $K \in \mathbb{N}$,

where $K$ is the order of the truncation. The IR perturbative expansion (24) is asymptotically of Gevrey-1 class (see discussion in Sec. III D of Ref. [31]). Thus, the asymptotic form of the coefficients $u_{1, k}^{(0)}$ entering in Eq. (24) are

$$
\left|u_{1, k}^{(\mathbf{0})}\right| \sim M\left(\hat{\theta}_{0}\right) S^{-k+\beta_{1}} \Gamma\left(k-\beta_{1}\right) \quad \text { as } \quad k \rightarrow+\infty,
$$

where $M\left(\hat{\theta}_{0}\right)=M_{0} \hat{\theta}_{0}^{\beta_{1}}\left(M_{0} \in \mathbb{R}\right)$ is an overall factor that depends on the angle $\hat{\theta}_{0}$ defined in the Borel plane. The function $K_{\mathrm{op}}(w)$ which optimizes the error between the asymptotic series and the exact solution can be calculated by evaluating the convergence rate as follows:

$$
\begin{gathered}
\left|u_{1, k}^{(\mathbf{0})}\right| w^{-k}>\left|u_{1, k+1}^{(\mathbf{0})}\right| w^{-k-1} \Rightarrow k<S w+\beta_{1} \\
\Rightarrow K_{\mathrm{op}}(w)=\left\lfloor S w+\beta_{1}\right\rfloor,
\end{gathered}
$$

where $\lfloor\bullet\rfloor$ is the floor function. Thus, the induced error $R_{K}(w)$ in the truncated expansion (B1) is

$$
\begin{aligned}
& R_{K_{\mathrm{op}}}(w) \\
& \sim M\left(\hat{\theta}_{0}\right) S^{-\left(K_{\mathrm{op}}-\beta_{1}+1\right)} \Gamma\left(K_{o p}-\beta_{1}+1\right) w^{-\left(K_{\mathrm{op}}+1\right)} \\
& \approx M\left(\hat{\theta}_{0}\right) \Gamma(S w+1)(S w)^{-S w} S^{-1} w^{-\beta_{1}-1} \quad\left(K_{\mathrm{op}} \approx S w+\beta_{1}\right) \\
& \sim \sqrt{2 \pi} M\left(\hat{\theta}_{0}\right)(S w)^{-1 / 2} w^{-\beta_{1}} e^{-S w} \text { as } w \rightarrow+\infty,
\end{aligned}
$$

where we used Stirling's formula for the last line. Clearly, this estimate depends on the asymptotic behavior of $\left|u_{k}^{(\mathbf{0})}\right|$ (B2). In that case if $k<K_{\mathrm{op}}$ the error is small when $\left|u_{k}^{(\mathbf{0})}\right|$ behaves like in Eq. (B2) and depends explicitly on $w$. Thus, when adding higher orders $k>K_{\text {op }}$ to the truncated expansion (B1) the mismatch between transseries and the exact solution increases. The situation gets more worrisome when solving the general dynamical system (14). In this general case, the leading-order perturbative contribution to $c_{\ell}(\hat{\tau})=O\left(\hat{\tau}^{-\ell}\right)$ [31]. As a result, the disagreement between the multiparameter transseries and the numerical solutions of the Legendre moments $c_{\ell}(\hat{\tau})$ is larger and expected if $\ell>K_{\mathrm{op}}$. This was precisely what some of us observed and extensively discussed in Refs. [31,32].

\section{APPENDIX C: IR TRANSSERIES SOLUTIONS IN THE PROPER TIME $\tau$ VARIABLE}

In Secs. III and IV we studied in detail the resurgent properties of the nonlinear ODEs (23) in terms of the $w$ variable. However, one can also analyze the original dynamical system in terms of the variable $\tau$ as it was done for the RTA Boltzmann equation $[30,31]$ for the detail. For completeness we present this analysis in this section.

The IR fixed points of the Legendre moments are $\bar{c}_{\ell}=0 \forall \ell>0$, namely $c_{\ell}(\tau) \rightarrow 0$ as $\tau \rightarrow+\infty$. From Eq. (14a), one obtains the exact formal solution of $T(\tau)$, i.e.,

$$
\begin{aligned}
T(\tau) & =\sigma_{T}\left(\sigma_{T} \tau\right)^{-1 / 3} \exp \left[-\frac{1}{30} \int \frac{d \tau}{\tau} c_{1}(\tau)\right] \\
& =: \frac{\sigma_{T}}{\hat{\tau}^{1 / 2}}(1+\bar{T}(\hat{\tau})),
\end{aligned}
$$

where $\sigma_{T} \in \mathbb{R}^{+}$is the integration constant with dimensions of energy and $\hat{\tau}:=\left(\sigma_{T} \tau\right)^{2 / 3} \cdot{ }^{11}$ Notice that $\hat{\tau}$ is dimensionless and guarantees the scale invariance of $c_{\ell}$ in the $w$ coordinate. In addition, in the second line we expanded asymptotically the solution of $c_{1}$ while satisfying that $\bar{T}(\tau) \rightarrow 0$ as $\hat{\tau} \rightarrow+\infty$. By substituting the solution (C1) into Eq. (14b), one obtains

\footnotetext{
${ }^{11}$ The integration and the series expansions are commutative with each other up to the value of integration constant determined from a given initial condition in general. In this sense, the value of $\sigma_{T}$ in a transseries is individually determined for each fixed point from a given initial condition.
} 


$$
\begin{aligned}
\frac{d c_{\ell}(\hat{\tau})}{d \hat{\tau}}= & F_{\ell}(\mathbf{c}, \hat{\tau}) \\
F_{\ell}(\mathbf{c}, \hat{\tau})= & -\frac{3}{2 \hat{\tau}}\left[\mathfrak{A}_{\ell} c_{\ell+1}(\hat{\tau})+\left(\overline{\mathfrak{B}}_{\ell}-\frac{2}{15} c_{1}(\hat{\tau})\right) c_{\ell}(\hat{\tau})+\mathfrak{\mho}_{\ell} c_{\ell-1}(\hat{\tau})\right] \\
& -(1+\bar{T}(\hat{\tau}))\left[\Lambda(\ell) c_{\ell}(\hat{\tau})+\hat{\kappa} \sum_{m, n=1}^{|m-n| \leq \ell} \Omega_{\ell m n} c_{m}(\hat{\tau}) c_{n}(\hat{\tau})+\hat{\kappa} \sum_{n=1}^{+\infty} \frac{(2 \ell-1)(\ell+1)}{3(4 n+1)} c_{n}(\hat{\tau})^{2} c_{\ell}(\hat{\tau})\right] .
\end{aligned}
$$

It is straightforward to find that $c_{\ell}(\hat{\tau})=O\left(\hat{\tau}^{-\ell}\right)$ by considering the asymptotic behavior of the solutions of Eqs. (14). For instance, one obtains that $c_{1}$ and $\bar{T}$ behave asymptotically as follows:

$$
c_{1}(\hat{\tau}) \sim-\frac{3 \mathfrak{C}_{1}}{2 \Lambda(1) \hat{\tau}}=-\frac{4}{\Lambda(1) \hat{\tau}}, \quad \bar{T}(\hat{\tau}) \sim-\frac{1}{20} \int \frac{d \hat{\tau}}{\hat{\tau}} c_{1}(\hat{\tau}) \sim-\frac{1}{5 \Lambda(1) \hat{\tau}} .
$$

As we proceeded in Secs. III and IV we rewrote the nonlinear ODEs (C2) in a matrix form, i.e.,

$$
\frac{d \mathbf{c}}{d \hat{\tau}}=\mathbf{F}(\mathbf{c}, \hat{\tau})
$$

where

$$
\begin{gathered}
\mathbf{c}(\hat{\tau})=\left(c_{1}(\hat{\tau}), \ldots, c_{L}(\hat{\tau})\right)^{\top} \\
\mathbf{F}(\mathbf{c}, \hat{\tau})=-\frac{1}{\hat{\tau}}[\mathfrak{X}(\mathbf{c}) \mathbf{c}(\hat{\tau})+\mathbf{\Gamma}]-(1+\bar{T}(\hat{\tau}))[\Lambda+\mathfrak{Y}(\mathbf{c})+\mathfrak{Z}(\mathbf{c})] \mathbf{c}(\hat{\tau}) .
\end{gathered}
$$

The matrices entering into the previous expressions are defined in Eqs. (16a) and (19). In order to build the transmonomials we linearized the dynamical system (14) around the IR fixed point,

$$
\begin{aligned}
\frac{d \delta \mathbf{c}(\hat{\tau})}{d \hat{\tau}} & =\sum_{\ell=1}^{L} \frac{\partial \mathbf{F}(\mathbf{c}, \hat{\tau})}{\partial c_{\ell}} \delta c_{\ell}(\hat{\tau}) \\
& =-\left[\Lambda+\frac{1}{\hat{\tau}}\left(\overline{\mathfrak{X}}-\frac{4 \overline{\mathfrak{Y}}}{\Lambda(1)}-\frac{\Lambda}{5 \Lambda(1)}\right)\right] \delta \mathbf{c}(\hat{\tau})-\frac{1}{\hat{\tau}} \delta \mathfrak{\vartheta}\left(\begin{array}{c}
c_{1}(\hat{\tau}) \\
0 \\
\vdots \\
0
\end{array}\right)+O\left(\mathbf{c} \delta \mathbf{c} / \hat{\tau}, \delta \mathbf{c} / \hat{\tau}^{2}\right) \\
& =-\left[\Lambda+\frac{1}{\hat{\tau}} \overline{\mathfrak{Y}}\right] \delta \mathbf{c}(\hat{\tau})+O\left(\mathbf{c} \delta \mathbf{c} / \hat{\tau}, \delta \mathbf{c} / \hat{\tau}^{2}\right),
\end{aligned}
$$

where

$$
\begin{gathered}
\overline{\mathfrak{W}}:=\overline{\mathfrak{X}}-\frac{4}{\Lambda(1)}\left(2 \overline{\mathfrak{Y}}+\frac{\Lambda}{20}\right), \\
(\delta \mathbf{c})=\hat{\kappa}\left(\begin{array}{cccc}
\sum_{m=1}^{2} \Omega_{1 m 1} \delta c_{m}(\hat{\tau}) & 0 & \cdots & 0 \\
\vdots & \vdots & \vdots \\
\sum_{m=\ell-1}^{\ell+1} \Omega_{\ell m 1} \delta c_{m}(\hat{\tau}) & 0 & \cdots & 0 \\
\vdots & \vdots & \vdots \\
\sum_{m=L-1}^{L+1} \Omega_{L m 1} \delta c_{m}(\hat{\tau}) & 0 & \cdots & 0
\end{array}\right), \quad \overline{\mathfrak{Y}}=\hat{\kappa}\left(\begin{array}{ccccc}
\Omega_{111} & \Omega_{112} & & \\
\Omega_{211} & \Omega_{212} & \Omega_{213} & & \\
& \ddots & \ddots & \ddots & \\
& & \Omega_{L-11 L-2} & \Omega_{L-11 L-1} & \Omega_{L-11 L} \\
& \Omega_{L 1 L-1} & \Omega_{L 1 L}
\end{array}\right) .
\end{gathered}
$$


In order to find the behavior of the fluctuations close to the IR fixed points it is needed to solve the eigenproblem for the matrix $\overline{\mathfrak{B}}$. Following Costin's prescription [54] we introduce the matrix $V$ as follows:

$$
\delta \tilde{\mathbf{c}}:=\tilde{V}(\hat{\tau}) \delta \mathbf{c}, \quad \tilde{V}(\hat{\tau}):=\mathbf{1}_{L}+V \hat{\tau}^{-1} .
$$

Thus, the linearized equation Eq. (C6) is modified as

$$
\begin{aligned}
\frac{d \delta \tilde{\mathbf{c}}}{d \hat{\tau}} & =-\tilde{V}(\hat{\tau})\left[\Lambda+\frac{1}{\hat{\tau}} \overline{\mathfrak{Y}}\right] \tilde{V}(\hat{\tau})^{-1} \delta \tilde{\mathbf{c}}(\hat{\tau})-V \tilde{V}(\hat{\tau})^{-1} \hat{\tau}^{-2} \delta \tilde{\mathbf{c}} \\
& =-\left[\Lambda+\frac{1}{\hat{\tau}}(\overline{\mathfrak{W}}+[V, \Lambda])\right] \delta \tilde{\mathbf{c}}(\hat{\tau})+O\left(\delta \tilde{\mathbf{c}} / \hat{\tau}^{2}\right),
\end{aligned}
$$

where the commutator between two matrices is denoted as $[A, B]:=A B-B A$. One can take diag. $(V)=(0, \ldots, 0)$, and the other components can be chosen such that

$$
\overline{\mathfrak{Y}}+[V, \Lambda]: \mapsto \hat{\mathfrak{W}}:=\operatorname{diag} .\left(\mathfrak{w}_{1}, \ldots, \mathfrak{w}_{L}\right) .
$$

Therefore, the solution of Eq. (C9) is given by

$$
\begin{aligned}
\delta \tilde{c}_{\ell}(\hat{\tau}) & =\sigma_{\ell} \frac{e^{-\Lambda(\ell) \hat{\tau}}}{\hat{\tau}^{\mathbf{w}_{\ell}}} \Rightarrow \delta c_{\ell}(\hat{\tau}) \\
& =\sigma_{\ell} \frac{e^{-\Lambda(\ell) \hat{\tau}}}{\hat{\tau}^{\mathbf{w}_{\ell}}}+\mathcal{O}\left(e^{-\Lambda(\ell) \hat{\tau}} / \hat{\tau}^{\mathfrak{w}_{\ell}+1}\right) .
\end{aligned}
$$

From the solution of the linearized fluctuations, the transseries takes the following form:

$$
\begin{aligned}
c_{\ell}(\tau) & =\sum_{|\mathbf{n}|=0}^{+\infty} \sum_{k=0}^{+\infty} u_{\ell, k}^{(\mathbf{n})} \Phi_{k}^{\mathbf{n}}, \\
\Phi_{k}^{\mathbf{n}} & :=\left(\prod_{j=1}^{L} \zeta_{j}^{n_{j}}\right) \hat{\tau}^{-k}, \quad \zeta_{j}:=\sigma_{j} \frac{e^{-S_{j} \hat{\tau}}}{\hat{\tau}^{\beta_{j}}},
\end{aligned}
$$

where $\mathbf{n} \in \mathbb{N}_{0}^{L}, \sigma_{i} \in \mathbb{C}$ is the integration constant, and $L \in \mathbb{N}$ is the truncation order of $\ell$, namely $1 \leq \ell \leq L$.

\section{APPENDIX D: ON THE EXISTENCE OF THE LYAPUNOV FUNCTIONAL}

In this section we present a proof of the existence of a Lyapunov function for the dynamical system of ODEs (22). Roughly speaking, Lyapunov functions are positive definite functions which are monotonically decreasing along the trajectories of the flows in phase space. These functions are used to determine the stability properties of ODEs and PDEs. In the RG flow approach to dynamical systems the Lyapunov function plays an analogous role of the $c$ function in QFT and/or the dynamical effective potential in non-Newtonian mechanics. For a nonautonomous dynamical system, the existence of the Lyapunov function allows us to identify the dynamical system as a RG flow from the global point of view.

In our approach the existence of the Lyapunov function is inferred directly from the dynamical system of ODEs (22). First, we promote this nonautonomous dynamical system to an autonomous one of one dimension higher by introducing an ODE for $w$ in terms of a new flow time $\rho$ as follows:

$$
\begin{gathered}
\frac{d \mathbf{c}(\rho)}{d \log \rho}=\beta(\mathbf{c}(\rho), \quad w(\rho)) \equiv-\frac{\partial \mathcal{V}}{\partial c_{i}}, \\
\frac{d w(\rho)}{d \log \rho}=\beta_{w}(w(\rho)) \equiv-\frac{\partial \mathcal{V}}{\partial w},
\end{gathered}
$$

where in the rhs of the previous expressions we introduce a positive definite differentiable function $\mathcal{V}$. It is straightforward to show that the function $\mathcal{V}$ decreases monotonically, i.e.,

$$
\begin{aligned}
\frac{d \mathcal{V}(\mathbf{c}(\rho), w(\rho))}{d \log \rho} & =\sum_{i=1}^{L} \frac{d c_{i}}{d \log \rho} \cdot \frac{\partial \mathcal{V}(\mathbf{c}(\rho), w(\rho))}{\partial c_{i}(\rho)}+\frac{d w(\rho)}{d \log \rho} \cdot \frac{\partial \mathcal{V}(\mathbf{c}(\rho), w(\rho))}{\partial w(\rho)}, \\
& =-\left[\sum_{i=1}^{L}\left|\beta_{i}(\mathbf{c}(\rho), w(\rho))\right|^{2}+\left|\beta_{w}(\mathbf{c}(\rho), w(\rho))\right|^{2}\right] \leq 0 .
\end{aligned}
$$

Therefore, $\mathcal{V}$ satisfies the properties required to be a candidate for the global Lyapunov function of the dynamical system (22) [see Eq. (B9) in Ref. [31]]. Although we have proven its existence for the studied case here, it is in general extremely difficult to calculate the exact Lyapunov function (cf. [29]) and this is beyond the scope of this work.

\section{APPENDIX E: EXISTENCE OF THE FORWARD ATTRACTOR AND HIGHER NONLINEARITIES OF TEH COLLISIONAL KERNAL}

In this section we outline the generic conditions that ensure the existence of a nonequilibrium forward attractor for systems undergoing Bjorken expansion described by 
relativistic kinetic theory within different approximations for the collisional kernel. Right now we have strong numerical evidence of this type of attractor for systems undergoing Bjorken expansion described microscopically within the relativistic kinetic theory framework and for different types of collisional kernels $[12,45,48,58,59,63$, 94,96,99,100,109-118].

Let us assume that the distribution function can be expanded around the thermal equilibrium while the deviations from this state are written in terms of a set of Legendre moments like in Eq. (8). The conservation law is the same for any physical system regardless of the collisional kernel (14a). On the other hand, the dynamical system of ODEs for the Legendre moments must satisfy certain symmetries given the constraints of the Bjorken flow. In this case, the dilation $\tau \rightarrow \gamma \tau$ (being $\gamma$ a constant) together with the conformal dimensionality of the Legendre moments and the temperature $\left(\Delta_{\mathbf{c}}, \Delta_{T}\right)=(0,1)$ restricts strongly the form of the differential equation. In addition, and given the results outlined in Fig. 7, one can assume that the small $\tau$ limit is dominated by singular terms which go like $\mathcal{O}\left(\tau^{-1}\right)$ such that the collision of kernel is $\mathcal{O}\left(\tau^{0}\right)$. Thus, the generic equation which satisfies these constraints is written generically as ${ }^{12}$

$$
\begin{gathered}
\frac{d \mathbf{c}(\tau)}{d \tau}=\mathbf{F}(\mathbf{c}, \tau), \\
\mathbf{F}(\mathbf{c}, \tau)=-\frac{1}{\tau}[\mathfrak{X}(\mathbf{c}) \mathbf{c}(\tau)+\Gamma]-\mathbf{G}(\mathbf{c}, T, \tau),
\end{gathered}
$$

where the terms entering into the matrix operator $\mathfrak{X}(\mathbf{c}) \mathbf{c}(\tau)+\Gamma$ are given by Eqs. (16a) and (18b), respectively, while $\mathbf{G}(\mathbf{c})$ is a generic function determined uniquely by the collisional kernel. This function depends on the moments, proper time, and temperature and its generic form must respect the symmetry restrictions mentioned above. Thus, the following general form of $\mathbf{G}(\mathbf{c})$ is

$\mathbf{G}(\mathbf{c})=\sum_{h=0}^{H} \sum_{n=1}^{N} \sum_{\ell_{1} \geq \cdots \geq \ell_{n}=1}^{L} \mathbf{g}_{h, n}^{\left(\ell_{1}, \ldots, \ell_{n}\right)} T^{h+1} \tau^{h} \prod_{n^{\prime}=1}^{n} c_{\ell_{n^{\prime}}}$,

\footnotetext{
${ }^{12}$ If one can consider a more general ansatz which encodes information about the high energy tails like the one taken in Ref. [45], one gets a set of nonlinear ODEs which mathematically resembles our general ODE (E1).
}

where $H \in \mathbb{N}_{0}$ and $N \in \mathbb{N}$. In addition, $g_{\ell, h, n}^{\left(\ell_{1}, \ldots, \ell_{n}\right)} \in \mathbb{R}$ is a dimensionless coefficient. ${ }^{13}$ The dynamical system of ODEs for the RTA Boltzmann (21) and FPE (14b) cases are a particular case of the previous expression.

Our transasymptotic analysis discussed in this work and in Refs. [29-32] shows that the existence of the forward attractor is ensured if the following conditions are satisfied:

(1) If the fixed point equation for the long-time limit given by

$$
\sum_{n=1}^{N} \sum_{\ell_{1} \geq \cdots \geq \ell_{n}=1}^{L} \mathbf{g}_{H, n}^{\left(\ell_{1}, \ldots, \ell_{n}\right)} \prod_{n^{\prime}=1}^{n} \bar{c}_{\ell_{n^{\prime}}}=0
$$

has a trivial solution, i.e., $\overline{\mathbf{c}}=\mathbf{0}$, then the IR fixed point corresponds to the local thermal equilibrium state.

(2) If the eigenvalues of the matrix $\mathbf{g}_{H, 1}^{\left(\ell_{1}\right)}$, around $\overline{\mathbf{c}}=\mathbf{0}$ are all positive, then the fixed point gives a (local) forward attractor.

(3) In case there is a subset of either exactly vanishing or positive eigenvalues of the matrix $\mathbf{g}_{H, 1}^{\left(\ell_{1}\right)}$, then there exists a sub(local)forward attractor $\mathfrak{M}$ in the phase space of dynamical variables $(\mathbf{c}, T, \tau)$ space. In this case, $\operatorname{dim}[\mathfrak{M}]=L+2-p$ where $p$ is the number of zeros or negative eigenvalues. In the $(\mathbf{c}, w)$ space, $\operatorname{dim}[\mathfrak{\mathfrak { U }}]=L+1-p$.

(4) In addition to the previous conditions, if $H=1$, then the transmonomials in the transseries are of the form (31b) like in the RTA and FPE cases, respectively. On the other hand, the pullback attractor will be determined by the term $\mathcal{O}\left(\tau^{-1}\right)$ in Eq. (E1). Notice however that in the most general case it is not necessarily ensured that there is only one invariant flow (generally dubbed as "attractor solution") that connects the UV and IR. A more careful analysis needs to be made by considering techniques based on Morse theory, cf. [32], and center manifolds [38,39] if there are vanishing eigenvalues for the collisional kernel.

\footnotetext{
${ }^{13} \mathbf{g}_{H, 1}^{\left(\ell_{1}\right)}$ is the linearized version of the collisional kernel in the eigenmodes and it corresponds to an $L$-by- $L$ matrix of the form

$$
\mathbf{g}_{H, 1}^{\left(\ell_{1}\right)}=\left(\begin{array}{ccc}
g_{1, H, 1}^{(1)} & \cdots & g_{1, H, 1}^{(L)} \\
\vdots & \ddots & \vdots \\
g_{L, H, 1}^{(1)} & \cdots & g_{L, H, 1}^{(L)}
\end{array}\right)
$$


[1] S. Chatrchyan et al. (CMS Collaboration), Phys. Lett. B 724, 213 (2013).

[2] A. Adare et al. (PHENIX Collaboration), Phys. Rev. Lett. 111, 212301 (2013).

[3] G. Aad et al. (ATLAS Collaboration), Phys. Rev. Lett. 110, 182302 (2013).

[4] B. Abelev et al. (ALICE Collaboration), Phys. Lett. B 719, 29 (2013).

[5] C. Aidala et al. (PHENIX Collaboration), Nat. Phys. 15, 214 (2019).

[6] C. Gale, S. Jeon, and B. Schenke, Int. J. Mod. Phys. A 28, 1340011 (2013).

[7] U. Heinz and R. Snellings, Annu. Rev. Nucl. Part. Sci. 63, 123 (2013).

[8] M. Bluhm, J. Hou, and T. Schäfer, Phys. Rev. Lett. 119, 065302 (2017).

[9] M. Bluhm and T. Schaefer, Phys. Rev. Lett. 116, 115301 (2016).

[10] M. Bluhm and T. Schäfer, Phys. Rev. A 92, 043602 (2015).

[11] T. Schäfer, Phys. Rev. A 94, 043644 (2016).

[12] J. Berges, M.P. Heller, A. Mazeliauskas, and R. Venugopalan, arXiv:2005.12299.

[13] W. Florkowski, M. P. Heller, and M. Spalinski, Rep. Prog. Phys. 81, 046001 (2018).

[14] P. Romatschke and U. Romatschke, Relativistic Fluid Dynamics in and out of Equilibrium, Cambridge Monographs on Mathematical Physics (Cambridge University Press, Cambridge, England, 2019).

[15] M. P. Heller and M. Spalinski, Phys. Rev. Lett. 115, 072501 (2015).

[16] W. Israel and J. M. Stewart, Ann. Phys. (N.Y.) 118, 341 (1979).

[17] I. Aniceto, B. Meiring, J. Jankowski, and M. Spaliński, J. High Energy Phys. 02 (2019) 073.

[18] M. Spaliński, Phys. Lett. B 784, 21 (2018).

[19] M. Spaliński, Phys. Lett. B 776, 468 (2018).

[20] A. Buchel, M. P. Heller, and J. Noronha, Phys. Rev. D 94, 106011 (2016).

[21] M. P. Heller, A. Kurkela, M. Spaliński, and V. Svensson, Phys. Rev. D 97, 091503 (2018).

[22] I. Aniceto and M. Spaliński, Phys. Rev. D 93, 085008 (2016).

[23] G. Basar and G. V. Dunne, Phys. Rev. D 92, 125011 (2015).

[24] J. Casalderrey-Solana, N. I. Gushterov, and B. Meiring, J. High Energy Phys. 04 (2018) 042.

[25] P. Romatschke, Phys. Rev. Lett. 120, 012301 (2018).

[26] J.-P. Blaizot and L. Yan, arXiv:2006.08815.

[27] P. Romatschke, Eur. Phys. J. C 77, 21 (2017).

[28] R. D. Weller and P. Romatschke, Phys. Lett. B 774, 351 (2017).

[29] A. Behtash, C. Cruz-Camacho, and M. Martinez, Phys. Rev. D 97, 044041 (2018).

[30] A. Behtash, C. Cruz-Camacho, S. Kamata, and M. Martinez, Phys. Lett. B 797, 134914 (2019).

[31] A. Behtash, S. Kamata, M. Martinez, and H. Shi, Phys. Rev. D 99, 116012 (2019).

[32] A. Behtash, S. Kamata, M. Martinez, and H. Shi, J. High Energy Phys. 07 (2020) 226.

[33] J. Bjorken, Phys. Rev. D 27, 140 (1983).
[34] M. P. Heller, R. Jefferson, M. Spaliński, and V. Svensson, Phys. Rev. Lett. 125, 132301 (2020).

[35] S. S. Gubser, Phys. Rev. D 82, 085027 (2010).

[36] S. S. Gubser and A. Yarom, Nucl. Phys. B846, 469 (2011).

[37] G. S. Denicol and J. Noronha, arXiv:1608.07869.

[38] T. Caraballo and X. Han, Applied Nonautonomous and Random Dynamical Systems: Applied Dynamical Systems, Springer Briefs in Mathematics (Springer International Publishing, New York, 2017).

[39] P. Kloeden and M. Rasmussen, Nonautonomous Dynamical Systems, Mathematical Surveys and Monographs (American Mathematical Society, Providence, 2011).

[40] A. H. Mueller, Phys. Lett. B 475, 220 (2000).

[41] A. H. Mueller, Nucl. Phys. B572, 227 (2000).

[42] J. Bjoraker and R. Venugopalan, Phys. Rev. C 63, 024609 (2001).

[43] J. Hong and D. Teaney, Phys. Rev. C 82, 044908 (2010).

[44] Y. Mehtar-Tani, Nucl. Phys. A966, 241 (2017).

[45] J.-P. Blaizot and N. Tanji, Nucl. Phys. A992, 121618 (2019).

[46] J.-P. Blaizot, J. Liao, and L. McLerran, Nucl. Phys. A920, 58 (2013).

[47] J.-P. Blaizot, B. Wu, and L. Yan, Nucl. Phys. A930, 139 (2014).

[48] N. Tanji and R. Venugopalan, Phys. Rev. D 95, 094009 (2017).

[49] G. D. Moore and D. Teaney, Phys. Rev. C 71, 064904 (2005).

[50] H. Grad, Commun. Pure Appl. Math. 2, 331 (1949).

[51] J. P. Boyd, Acta Appl. Math. 56, 1 (1999).

[52] O. Costin, Asymptotics and Borel Summability, Monographs and Surveys in Pure and Applied Mathematics (CRC Press, Boca Raton, FL, 2008).

[53] O. Costin, Int. Math. Res. Not. 1995, 377 (1995).

[54] O. Costin, Duke Math. J. 93, 289 (1998).

[55] S. Gukov, J. High Energy Phys. 01 (2016) 020.

[56] S. Gukov, Nucl. Phys. B919, 583 (2017).

[57] A. Kurkela, W. van der Schee, U. A. Wiedemann, and B. Wu, Phys. Rev. Lett. 124, 102301 (2020).

[58] S. Jaiswal, C. Chattopadhyay, A. Jaiswal, S. Pal, and U. Heinz, Phys. Rev. C 100, 034901 (2019).

[59] J.-P. Blaizot and L. Yan, Ann. Phys. (Amsterdam) 412, 167993 (2020).

[60] P. B. Arnold, G. D. Moore, and L. G. Yaffe, J. High Energy Phys. 01 (2003) 030.

[61] E. Lifshitz and L. Pitaevskii, Physical Kinetics (Pergamon Press, Oxford, 1981).

[62] M. M. Le Bellac, Thermal Field Theory (Cambridge University Press, Cambridge, England, 2000).

[63] J.-P. Blaizot and L. Yan, J. High Energy Phys. 11 (2017) 161.

[64] G. Baym, H. Monien, C. Pethick, and D. Ravenhall, Phys. Rev. Lett. 64, 1867 (1990).

[65] H. Heiselberg, Phys. Rev. D 49, 4739 (1994).

[66] M. C. A. York, A. Kurkela, E. Lu, and G. D. Moore, Phys. Rev. D 89, 074036 (2014).

[67] D. Bazow, G. Denicol, U. Heinz, M. Martinez, and J. Noronha, Phys. Rev. Lett. 116, 022301 (2016).

[68] D. Bazow, G. Denicol, U. Heinz, M. Martinez, and J. Noronha, Phys. Rev. D 94, 125006 (2016). 
[69] M. Krook and T. T. Wu, Phys. Rev. Lett. 36, 1107 (1976).

[70] A. Bobylev, Sov. Phys. Dokl. 20, 820 (1976).

[71] E. Molnar, H. Niemi, and D. Rischke, Phys. Rev. D 93, 114025 (2016).

[72] M. Martinez, M. McNelis, and U. Heinz, Phys. Rev. C 95, 054907 (2017).

[73] M. Martinez and M. Strickland, Phys. Rev. C 79, 044903 (2009).

[74] G. Denicol, T. Koide, and D. Rischke, Phys. Rev. Lett. 105, 162501 (2010).

[75] W. Florkowski, R. Ryblewski, and M. Strickland, Nucl. Phys. A916, 249 (2013).

[76] W. Florkowski, R. Ryblewski, and M. Strickland, Phys. Rev. C 88, 024903 (2013).

[77] G. S. Denicol, J. Noronha, H. Niemi, and D. H. Rischke, Phys. Rev. D 83, 074019 (2011).

[78] D. Teaney and L. Yan, Phys. Rev. C 89, 014901 (2014).

[79] C. Chattopadhyay, A. Jaiswal, S. Pal, and R. Ryblewski, Phys. Rev. C 91, 024917 (2015).

[80] R. S. Bhalerao, A. Jaiswal, S. Pal, and V. Sreekanth, Phys. Rev. C 89, 054903 (2014).

[81] A. Jaiswal, Phys. Rev. C 88, 021903 (2013).

[82] A. Jaiswal, Phys. Rev. C 87, 051901 (2013).

[83] P. Romatschke, Phys. Rev. D 85, 065012 (2012).

[84] L. Yan, J. Phys. Conf. Ser. 389, 012014 (2012).

[85] G. Denicol, E. Molnár, H. Niemi, and D. Rischke, Eur. Phys. J. A 48, 170 (2012).

[86] R. Baier, P. Romatschke, D. T. Son, A. O. Starinets, and M. A. Stephanov, J. High Energy Phys. 04 (2008) 100.

[87] O. Costin and R. Costin, Inventiones Mathematicae 145, 425 (2001).

[88] S. Lang, Complex Analysis, Graduate Texts in Mathematics (Springer, New York, 2010).

[89] H. Heiselberg and A.-M. Levy, Phys. Rev. C 59, 2716 (1999).

[90] A. Kurkela, U. A. Wiedemann, and B. Wu, Eur. Phys. J. C 79, 965 (2019).

[91] A. Kurkela, U. A. Wiedemann, and B. Wu, Eur. Phys. J. C 79, 759 (2019).

[92] N. Borghini and C. Gombeaud, Eur. Phys. J. C 71, 1612 (2011).

[93] P. Romatschke, Eur. Phys. J. C 78, 636 (2018).

[94] J.-P. Blaizot and L. Yan, Phys. Lett. B 780, 283 (2018).

[95] S. Kamata, M. Martinez, P. Plaschke, S. Ochsenfeld, and S. Schlichting, Phys. Rev. D 102, 056003 (2020).
[96] G. Giacalone, A. Mazeliauskas, and S. Schlichting, Phys. Rev. Lett. 123, 262301 (2019).

[97] S. Schlichting and D. Teaney, Annu. Rev. Nucl. Part. Sci. 69, 447 (2019).

[98] K. Dusling, G. D. Moore, and D. Teaney, Phys. Rev. C 81, 034907 (2010).

[99] A. Kurkela, A. Mazeliauskas, J.-F. Paquet, S. Schlichting, and D. Teaney, Phys. Rev. Lett. 122, 122302 (2019).

[100] A. Kurkela, A. Mazeliauskas, J.-F. Paquet, S. Schlichting, and D. Teaney, Phys. Rev. C 99, 034910 (2019).

[101] https://pub.uni-bielefeld.de/record/2939684

[102] D. Bazow, U. W. Heinz, and M. Martinez, Phys. Rev. C 91, 064903 (2015).

[103] G. S. Denicol, U. W. Heinz, M. Martinez, J. Noronha, and M. Strickland, Phys. Rev. D 90, 125026 (2014).

[104] G. S. Denicol, U. W. Heinz, M. Martinez, J. Noronha, and M. Strickland, Phys. Rev. Lett. 113, 202301 (2014).

[105] L. Tinti, R. Ryblewski, W. Florkowski, and M. Strickland, Nucl. Phys. A946, 29 (2016).

[106] G. S. Denicol, W. Florkowski, R. Ryblewski, and M. Strickland, Phys. Rev. C 90, 044905 (2014).

[107] W. Florkowski, R. Ryblewski, M. Strickland, and L. Tinti, Phys. Rev. C 89, 054909 (2014).

[108] W. Florkowski, E. Maksymiuk, R. Ryblewski, and M. Strickland, Phys. Rev. C 89, 054908 (2014).

[109] M. Strickland, J. High Energy Phys. 12 (2018) 128.

[110] M. Strickland and U. Tantary, J. High Energy Phys. 10 (2019) 069.

[111] D. Almaalol, A. Kurkela, and M. Strickland, Phys. Rev. Lett. 125, 122302 (2020).

[112] A. Kurkela and G. D. Moore, J. High Energy Phys. 11 (2011) 120.

[113] A. Kurkela and Y. Zhu, Phys. Rev. Lett. 115, 182301 (2015).

[114] D. Almaalol, M. Alqahtani, and M. Strickland, Phys. Rev. C 99, 014903 (2019).

[115] D. Almaalol and M. Strickland, Phys. Rev. C 97, 044911 (2018).

[116] J. Berges, K. Boguslavski, S. Schlichting, and R. Venugopalan, Phys. Rev. D 89, 114007 (2014).

[117] J. Berges, K. Boguslavski, S. Schlichting, and R. Venugopalan, Phys. Rev. D 89, 074011 (2014).

[118] A. Mazeliauskas and J. Berges, Phys. Rev. Lett. 122, 122301 (2019). 